\title{
THE GOULD’S BELT VERY LARGE ARRAY SURVEY. II. THE SERPENS REGION
}

\author{
Gisela N. Ortiz-León ${ }^{1}$, Laurent Loinard ${ }^{1,2}$, Amy J. Mioduszewski ${ }^{3}$, Sergio A. DziB ${ }^{2}$, Luis F. Rodríguez ${ }^{1,4}$, \\ Gerardo Pech ${ }^{1}$, Juana L. Rivera ${ }^{1}$, Rosa M. Torres ${ }^{5}$, Andrew F. Boden ${ }^{6}$, Lee Hartmann ${ }^{7}$, Neal J. Evans II ${ }^{8}$, \\ Cesar Briceño ${ }^{9}$, John Tobin ${ }^{10,11}$, Marina A. Kounkel ${ }^{7}$, and Rosa A. González-Lópezlira ${ }^{1}$ \\ ${ }^{1}$ Centro de Radioastronomía y Astrofísica, Universidad Nacional Autónoma de Mexico, Morelia 58089, Mexico; g.ortiz@crya.unam.mx \\ ${ }^{2}$ Max Planck Institut für Radioastronomie, Auf dem Hügel 69, D-53121 Bonn, Germany \\ ${ }^{3}$ National Radio Astronomy Observatory, Domenici Science Operations Center, 1003 Lopezville Road, Socorro, NM 87801, USA \\ ${ }^{4}$ King Abdulaziz University, P.O. Box 80203, Jeddah 21589, Saudi Arabia \\ ${ }^{5}$ Instituto de Astronomía y Meteorología, Universidad de Guadalajara, Av. Vallarta 2602, Col. Arcos Vallarta, 44130, Guadalajara, Jalisco, México \\ ${ }^{6}$ Division of Physics, Math and Astronomy, California Institute of Technology, 1200 East California Boulevard, Pasadena, CA 91125, USA \\ ${ }_{7}$ Department of Astronomy, University of Michigan, 500 Church Street, Ann Arbor, MI 48105, USA \\ ${ }^{8}$ Department of Astronomy, The University of Texas at Austin, 2515 Speedway, Stop C1400, Austin, TX 78712-1205, USA \\ ${ }_{9}$ Cerro Tololo Interamerican Observatory, Casilla 603, La Serena, Chile \\ ${ }^{10}$ National Radio Astronomy Observatory, Charlottesville, VA 22903, USA \\ Received 2014 January 27; accepted 2015 March 12; published 2015 May 13
}

\section{ABSTRACT}

We present deep $(\sim 17 \mu \mathrm{Jy})$ radio continuum observations of the Serpens molecular cloud, the Serpens south cluster, and the W40 region obtained using the Very Large Array in its A configuration. We detect a total of 146 sources, 29 of which are young stellar objects (YSOs), 2 of which are BV stars, and 5 more of which are associated with phenomena related to YSOs. Based on their radio variability and spectral index, we propose that about 16 of the remaining 110 unclassified sources are also YSOs. For approximately 65\% of the known YSOs detected here as radio sources, the emission is most likely non-thermal and related to stellar coronal activity. As also recently observed in Ophiuchus, our sample of YSOs with X-ray counterparts lies below the fiducial Güdel \& Benz relation. Finally, we analyze the proper motions of nine sources in the W40 region. This allows us to better constrain the membership of the radio sources in the region.

Key words: astrometry - magnetic fields - radiation mechanisms: non-thermal - radio continuum: stars techniques: interferometric

\section{INTRODUCTION}

Radio continuum observations toward star-forming regions are relevant because they provide insights into thermal and non-thermal emission in young stellar objects (YSOs), stellar coronal activity of YSOs, and magnetic fields. Different processes are invoked to explain the origin of the radio emission in these different kinds of objects. Embedded Class I protostars have most often been detected as thermal bremsstrahlung sources, and this emission is predominantly due to collimated thermal winds or jets. In the case of more massive stars, the radio emission can also originate from optically thick or thin compact $\mathrm{H}$ II regions (Hughes 1988; Estalella et al. 1991; Gómez et al. 2000), or from ionized winds (Felli et al. 1998). Non-thermal (gyrosynchrotron) emission has also been detected in a number of sources. This mechanism produces radiation characterized by high brightness temperature, high variability, and often a negative spectral index and some level of circular polarization (e.g., Hughes 1991; Hughes et al. 1995; Garay et al. 1996). This non-thermal radio emission is generally present in more evolved YSOs (Class III sources), but it has also been detected in a number of Class II and even in a few Class I sources (e.g., Forbrich et al. 2007; Dzib et al. 2010; Deller et al. 2013). We note that very little has been done on the characterization of the Serpens, Serpens South, and W40 regions at radio wavelengths.

The Aquila rift/Serpens complex is one of the clouds selected for observations as part of The Gould's Belt Distance Survey, which is a large project designed to determine accurate

\footnotetext{
${ }^{11}$ Hubble Fellow
}

distances to stars in the most often studied star-forming sites (Loinard 2013). In this paper we report on new sensitive and high angular resolution radio observations of the Serpens, Serpens South, and W40 regions. This paper is the second (after that by Dzib et al. 2013, which dealt with the Ophiuchus region) in a series that will focus on the analysis of the radio emission from YSOs in the star-forming regions of the Gould's Belt using the Karl G. Jansky Very Large Array (JVLA). The observations cover large fields of view of the three regions: 900, 290, and 280 square arcminutes in Serpens, Serpens South, and W40, respectively, i.e., considerably larger than previous observations carried out at radio wavelengths toward these regions.

The Serpens molecular cloud has been studied via multiwavelength observations ever since it was recognized as an active star-forming region by Strom et al. (1974). The cloud belongs to a larger complex of local optically dark molecular clouds called the Aquila rift, which, in turn, is part of the Gould's Belt (e.g., Dame et al. 1987; Perrot \& Grenier 2003). The Serpens cloud shows large-scale irregular dark structures in optical images, and several nebulae can be distinguished. One of the most prominent is the Serpens nebula, which is illuminated by the pre-main-sequence (PMS) star SVS 2 (Strom et al. 1974, 1976; Worden \& Grasdalen 1974; King et al. 1983; Warren-Smith et al. 1987; Gomez de Castro et al. 1988). The region, about $6^{\prime}$ across centered on the Serpens nebula, is known as the Serpens cloud core and was described in early observations by Loren et al. (1979) as a nearly circular, high-density formaldehyde $\left(\mathrm{H}_{2} \mathrm{CO}\right)$ core. A more elongated structure extends in a north west/south east direction as seen, 
for instance, on maps of far-IR and $\mathrm{NH}_{3}$ emission (e.g., Torrelles et al. 1989). The Serpens core is populated by more than 300 objects found in many different evolutionary stages and coexisting within the central $\sim 0.5-0.7 \mathrm{pc}$ of the core (Eiroa et al. 2008 and references therein).

Near- and mid-infrared (IR) observations have identified a large number of Class II PMS stars, flat-spectrum sources, and Class I protostars embedded in the cloud core (Eiroa \& Casali 1992; Sogawa et al. 1997; Giovannetti et al. 1998; Kaas 1999; Kaas et al. 2004). Class 0 protostars and protostellar condensations have also been found by means of submillimeter, millimeter, and far-IR observations (Casali et al. 1993; Hurt \& Barsony 1996; Testi \& Sargent 1998). In addition, some of these YSOs have X-ray counterparts (Preibisch 1998, 2003, 2004; Giardino et al. 2007). Spitzer/IRAC and MIPS observations extend to a larger region of $\sim 6-9$ pc (Harvey et al. 2006, 2007a, 2007b; Oliveira et al. 2010). Briefly, these most recent works identified at least two main centers of star formation, which were named Clusters A and B. Cluster A is the already described region referred to as the Serpens core, while Cluster B, $\sim 35^{\prime}$ to the south of Cluster $\mathrm{A}$ and also referred to as Serpens G3-G6, was not observed by us. At radio wavelengths, the Serpens core has been observed by Eiroa et al. (2005) with the Very Large Array (VLA). A total of 22 radio continuum sources were detected, 16 of which were proposed to be associated with Class 0, Class I, flat spectrum, and Class II YSOs of the core. AMI Consortium et al. (2012) carried out deep radio continuum observations at $1.8 \mathrm{~cm}$ with the Arcminute Microkelvin Imager Large Array (providing an angular resolution of $\sim 30 \mathrm{arcsec}$ ) of the 19 protostellar cores reported in the Spitzer catalog of Dunham et al. (2008). They detected eight radio sources, six possibly associated with deeply embedded YSOs.

At an angular distance of $\sim 3^{\circ}$ to the south of the center of the Serpens core lies the star-forming region known as W40. Assuming a distance of $415 \mathrm{pc}$ (see discussion below), this corresponds to a spatial distance of $\sim 22 \mathrm{pc}$. Three main components are recognized in this region. First is the cold molecular cloud G28.8+3.5 (Goss \& Shaver 1970) with an extent of $\sim 1 \mathrm{deg}$, centered around the smaller $\left(\sim 20^{\prime}\right)$ dense molecular core, TGU 279-P7 (Dobashi et al. 2005). Second, there is a blister $\mathrm{H}$ II region (W40) of diameter $\sim 6^{\prime}$ centered on J2000 coordinates $18^{\mathrm{h}} 30^{\mathrm{m}} 29^{\mathrm{s}},-2^{\circ} 05$ ! 4 and adjacent to the molecular cloud (Westerhout 1958). Finally, the W40 region hosts an embedded OB star cluster that is the primary excitation source for the W40 $\mathrm{H}$ II region (Smith et al. 1985). There is evidence for ongoing star formation from dense molecular material in this region, as suggested by the detection of an IRAS source, a cold ammonia core, and a number of millimeter-wave sources associated with the cluster (Molinari et al. 1996; Maury et al. 2011).

The stellar population of W40 has also been investigated through observations at different wavelengths. Crutcher \& Chu (1982) and Smith et al. (1985) found seven IR bright sources with optical counterparts behind 9-10 mag of visual extinction within the central $3^{\prime}$ of W40. According to the spectral energy distributions (SEDs) from the IR through the millimeter obtained by Smith et al. (1985) and Vallee \& MacLeod (1994), most of these bright sources are surrounded by significant amounts of circumstellar material. A cluster of near-IR sources is detected in the 2MASS images within the central 5' (Smith et al. 1985). Recently, Rodríguez et al. (2010) observed the W40 region at $3.6 \mathrm{~cm}$ with the VLA. They found a cluster of 20 compact sources in the central portion of the W40 IR cluster, many of which correspond to the known IR sources. The W40 cluster has also been observed with the Chandra X-ray Observatory. These observations reveal approximately 200 sources associated with the cluster, the majority of which are thought to be low-mass YSOs (Kuhn et al. 2010). More recently, Shuping et al. (2012) determined the spectral classification and SEDs of the brightest members of the central stellar cluster in the W40 region. They identified four main-sequence OB stars, two Herbig AeBe stars, and two low-mass YSOs (Class II).

Very close to W40 in projection on the plane of the sky lies an embedded cluster of IR sources referred to as Serpens South. This cluster was recently discovered by Gutermuth et al. (2008) from Spitzer observations of the Aquila rift region. They identified 54 sources classified as either Class I or flat SED and 37 Class II YSOs within a $14^{\prime} \times 10^{\prime}$ region.

Both clusters, Serpens South and W40, have been observed at 70-500 $\mu \mathrm{m}$ with the Herschel Space Telescope as part of the Gould Belt program (André et al. 2010; Bontemps et al. 2010; Könyves et al. 2010). The region observed toward the Aquila rift is $\sim 3: 3 \times 3: 3$ in size, with seven YSOs safely classified as Class 0 objects, only in the reduced area of Serpens South (Bontemps et al. 2010). Additionally, around 45 (for $\left.T_{\text {bol }}^{70-500}<27 \mathrm{~K}\right)$ and $60\left(L_{\text {submm }}^{\lambda>350} / L_{\text {bol }}^{70-500}>0.03\right)$ objects in the entire field of Aquila (W40, Serpens South, and the H II region Sh2-62) were proposed to be Class 0 YSOs. Maury et al. (2011) carried out a $1.2 \mathrm{~mm}$ dust continuum mapping of the Aquila complex with the MAMBO bolometer array on the IRAM $30 \mathrm{~m}$ telescope. Twenty-five continuum sources were identified in the Serpens South protocluster and their evolutionary stages were estimated, resulting in nine starless sources, nine Class 0, and seven Class 0/I protostars. In the W40 region, 35 sources were detected from these observations, separated into 14 starless, 8 Class 0, 4 Class 0/I, and 9 Class I YSOs.

Additionally, a larger number of YSOs have been identified in the Aquila/Serpens region by the c2d ("from Molecular cores to Planet-forming Disks") and GB ("Spitzer Gould Belt Survey") surveys (Dunham et al. 2013; L. E. Allen et al. 2015, in preparation). A total of 1524 YSOs with determined IR spectral indexes and belonging to these regions are reported in their catalogs.

Early estimates of the distance to the Serpens molecular cloud seemed to converge toward a value of $260 \mathrm{pc}$ (see the discussion in Eiroa et al. 2008). On the basis of radial velocity measurements from molecular line observations, Gutermuth et al. (2008) suggest that the Serpens South cluster is comoving with the Serpens Main embedded cluster $3^{\circ}$ to the north, and, therefore, that it should be part of Serpens. They also assign a distance of $260 \mathrm{pc}$ to the cluster, which corresponds to the distance to the front edge of the Aquila rift (Straižys et al. 1996). Based on spectral types determined for a few main-sequence stars in the W40 region, Shuping et al. (2012) estimated a distance to the W40 cluster of between 455 and $535 \mathrm{pc}$. Therefore, the W40 region and the Serpens South cluster (thought to be at $260 \mathrm{pc}$ ) are usually regarded as separate objects. On the other hand, Bontemps et al. (2010) argue that the W40 region, the Aquila rift, and Serpens Main are parts of the same star-forming region, located at a common distance of $260 \mathrm{pc}$. However, more recently, based on a comparison of the X-ray luminosity function of the Serpens 
Table 1

JVLA Observations

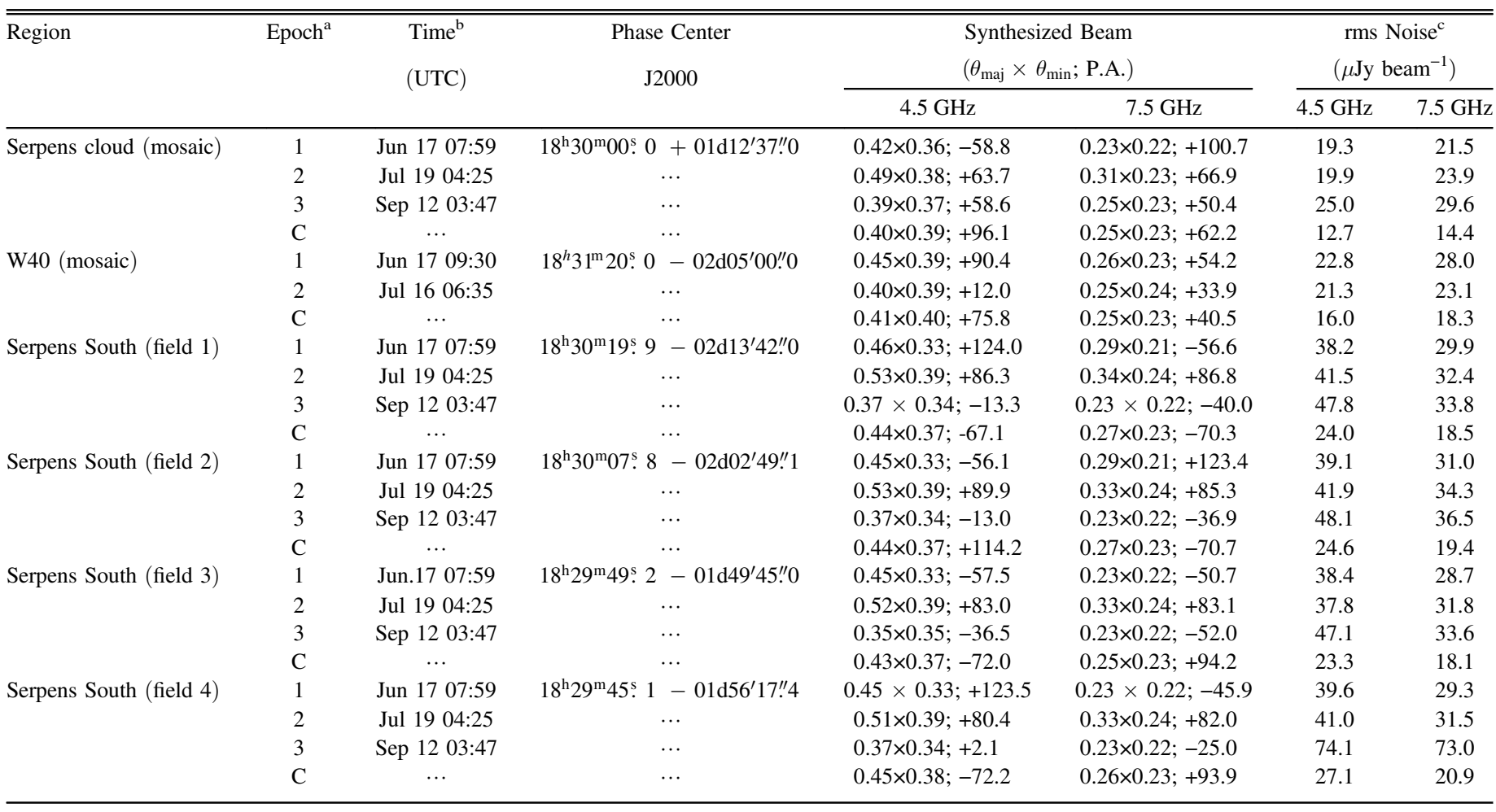

${ }^{\text {a }} \mathrm{C}$ indicates parameters measured in the images after combining the epochs.

${ }^{\mathrm{b}}$ Start time of the observing sessions. All epochs were observed in 2011.

${ }^{\mathrm{c}}$ Measured at the center of the Stokes $I$ image.

cluster with the previously published Orion Nebula cluster, Winston et al. (2010) obtained a new distance for the Serpens core of $360_{-13}^{+22} \mathrm{pc}$, while Dzib et al. (2010) claimed a distance of $415 \pm 5 \mathrm{pc}$ to the same, based on a VLBA parallax of the embedded young AeBe star EC95. Given that the estimation of Dzib et al. (2010) is the most recent and accurate, in this paper we adopt a distance of $415 \mathrm{pc}$ for the three regions in the Aquila complex, including Serpens, Serpens South, and W40.

The rest of this paper is organized as follows. In Section 2 we present details of the JVLA observations; in Section 3 we describe the results, which are analyzed and discussed in Sections 4 and 5. Section 6 is a summary of our results.

\section{OBSERVATIONS AND DATA REDUCTION}

The Serpens molecular cloud, the W40 region, and the Serpens South cluster were observed with the JVLA in its A configuration. Two frequency sub-bands, each $1 \mathrm{GHz}$ wide and centered at 4.5 and $7.5 \mathrm{GHz}$, respectively, were recorded simultaneously. The Serpens molecular cloud and the Serpens South cluster were observed in the same observing sessions on three different epochs (2011 June 17, July 19, and September $12 \mathrm{UT}$ ), using 25 and 4 pointings, respectively. The W40 region, on the other hand, was only observed on two epochs (2011 June 17 and July 16), using 13 pointings. This dualfrequency strategy was chosen to enable the characterization of the spectral index of the detected sources, while the multiepoch observations were aimed at determining the radio flux variability and helping in the identification of the emission mechanisms (thermal versus non-thermal). The details of the observations are listed in Table 1 . The 25 pointings were used to map an area of 900 (530) square arcminutes at 4.5 (7.5) $\mathrm{GHz}$ of the Serpens molecular cloud (see Figure 1). The covered area of the W40 region using the 13 pointings was 415 (280) square arcminutes at 4.5 (7.5) GHz. The four pointings used for the Serpens South cluster covered an area of 290 (110) square arcminutes at 4.5 (7.5) GHz (see Figure 1). The number of individual pointings observed toward the Serpens molecular cloud and the W40 region and the spacing between them were chosen to optimize the compromise between uniform sensitivity and inclusion of the largest possible number of known young stars.

3C 286 and J1804+010 were used as the standard flux and phase calibrator, respectively. The observations of the Serpens molecular cloud and the Serpens South cluster were carried out in $2 \mathrm{hr}$ scheduling blocks, while the observations of the W40 region in $1 \mathrm{hr}$ scheduling blocks. Each epoch consists of a 9-minute observation of the flux calibrator, followed by a series of two to four different target pointings (for 3 minutes each) bracketed by phase calibrator observations of 1 minute. Thus, 3 minutes were spent on each target field in each epoch. The data were edited and calibrated in a standard fashion using the Common Astronomy Software Applications package (CASA) version 3.4. Once calibrated, the data at each frequency were imaged (Stokes parameter $I$ ) using the CASA task clean. The 25 target pointings toward the Serpens molecular cloud were used to construct a mosaic of $22,400 \times 20,412$ pixels with a pixel size of 0.09 at $4.5 \mathrm{GHz}$. This was done by setting the imagermode parameter to "mosaic" in the clean task. For the W40 region, the 13 target pointings were used to obtain a mosaic of 15,552 × 18,522 pixels with a pixel size of 0.09 at 

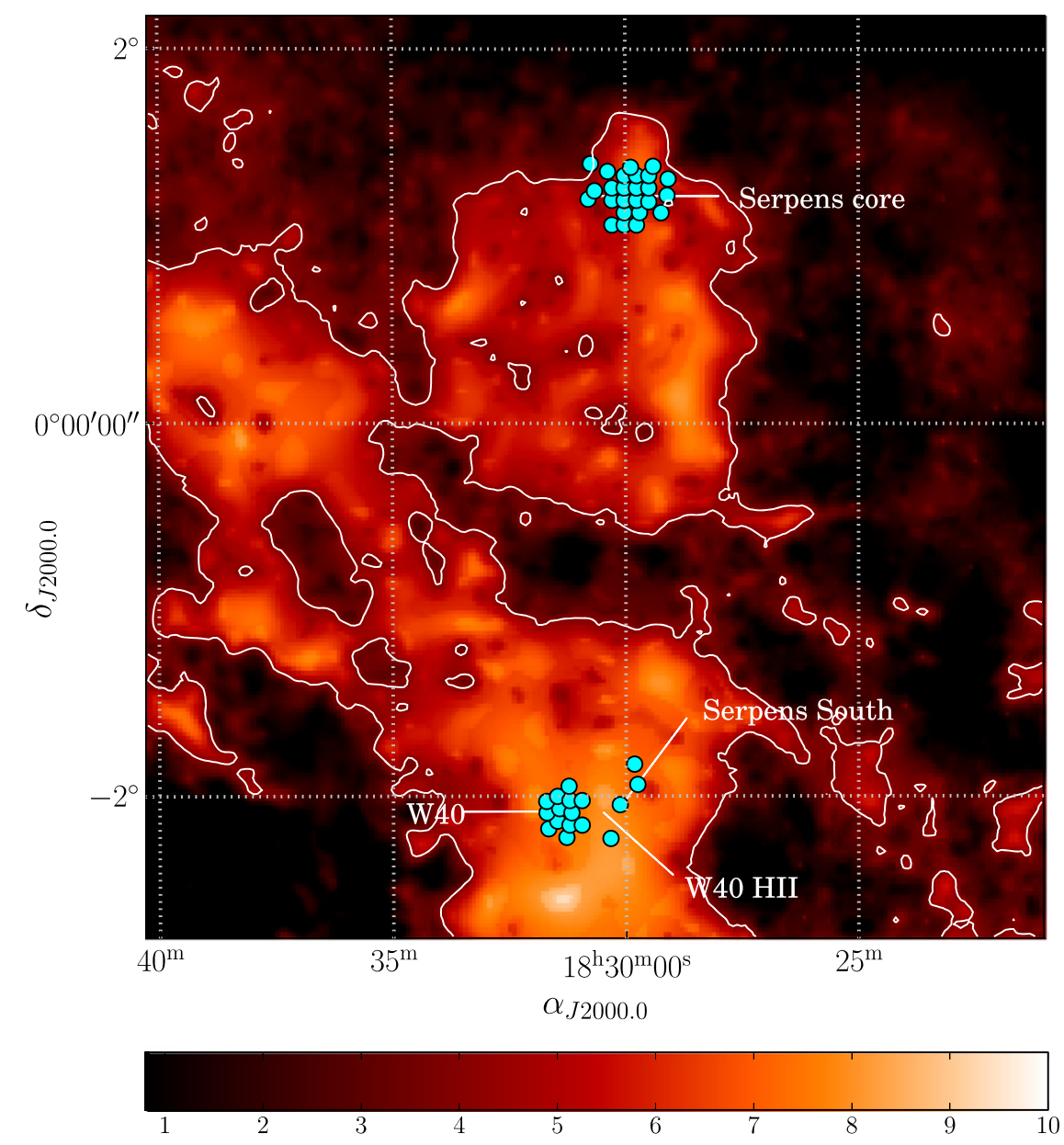

$\mathrm{A}_{V}$ (mag)

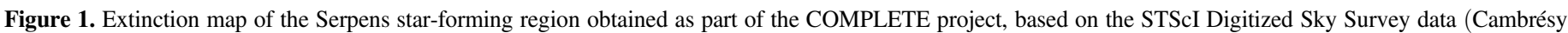
1999). The small cyan circles represent the size at $7.5 \mathrm{GHz}$ of the fields observed in each region.

4.5 GHz. The phase centers of the mosaics are indicated in Table 1. The four Serpens South cluster fields were imaged separately, using an image size of 6750 pixels in each dimension and a pixel size of $0 . .09$ at $4.5 \mathrm{GHz}$. The pixel size for the images at $7.5 \mathrm{GHz}$ was $0 . \prime 055$, and the number of pixels was adjusted to cover the same area as the images at $4.5 \mathrm{GHz}$. In order to take into account the non-coplanarity of the baselines far from the phase center, we set the gridmode parameter to "widefield" with wprojplanes $=128$ and facets $=1$. We also correct the images for the primary beam attenuation. The rms noise levels reached at each frequency and epoch are given in Table 1.

To produce images with improved sensitivity, the three or two epochs (in Serpens and W40, respectively) were combined and jointly imaged. The rms noise levels achieved after combining the epochs, as well as the synthesized beam (angular resolution) of the final maps, are also given in Table 1.

Gyrosynchrotron emission often (but not always) exhibits some level of circular polarization (Dulk 1985). To test for circular polarized radio emission, we produced images of the Stokes parameter $V$. The brightness distribution of the Stokes parameter $\mathrm{V}$ is obtained by applying the Fourier transform to the Stokes visibility function $V_{V}$, which is given by $V_{V}=1 / 2\left(V_{R R}-V_{L L}\right)$, where $V_{R R}$ is the correlation of the right circularly polarized responses and $V_{L L}$ is the correlation of those left circularly polarized.
We imaged the Stokes parameter $V$ for all 42 fields (25 of the Serpens molecular cloud, 4 of the Serpens South cluster, and 13 of the W40 region), separately at each frequency, and combined the three or two epochs. We did not apply any polarization correction in addition to the standard $6 \mathrm{~cm}$ continuum calibration. Also, in order to avoid beam squint (Dzib et al. 2013), only the inner quarter (in area) of the primary beam was tested, producing images of $4^{\prime} \times 4^{\prime}$ in size.

\section{RESULTS}

\subsection{Source Identification}

The identification of the radio sources was done through a visual inspection using the deep radio images. For the Serpens molecular cloud and the W40 region this means that we used the mosaics obtained after combining the epochs. For the Serpens South cluster we used the combined individual fields.

Once the sources were identified in those images, their fluxes and their positions at 4.5 and $7.5 \mathrm{GHz}$ were obtained by performing two-dimensional Gaussian fittings, using the CASA task imfit. The results of these fittings are listed in Table 2. Sources are named GBS-VLA Jhhmmss.ss—ddmmss.s, where GBS-VLA stands for Gould's Belt VLA Survey and hhmmss.ss - ddmmss.s are the coordinates of the source. We considered for the quoted flux densities in the table three sources of error: an error resulting from the statistical noise in the images, a 
Table 2

Radio Sources Detected in Serpens

\begin{tabular}{|c|c|c|c|c|c|c|}
\hline $\begin{array}{l}\text { GBS-VLA } \\
\text { Name/Position }\end{array}$ & $\begin{array}{c}\text { New } \\
\text { Detection? }^{\mathrm{a}}\end{array}$ & $\begin{array}{c}\text { Flux Density } \\
(4.5 \mathrm{GHz}) \\
(\mathrm{mJy})\end{array}$ & $\begin{array}{c}\text { Variability } \\
(4.5 \mathrm{GHz}) \\
(\%)\end{array}$ & $\begin{array}{c}\text { Flux Density } \\
(7.5 \mathrm{GHz}) \\
(\mathrm{mJy})\end{array}$ & $\begin{array}{c}\text { Variability } \\
(7.5 \mathrm{GHz}) \\
(\%)\end{array}$ & $\begin{array}{l}\text { Spectral } \\
\text { Index }\end{array}$ \\
\hline \multicolumn{7}{|c|}{ Detected Sources in the Serpens Molecular Cloud: } \\
\hline $\mathrm{J} 182850.71+011102.7$ & $\mathrm{Y}$ & $0.28 \pm 0.05$ & $38 \pm 19$ & $\cdots$ & $\cdots$ & $\cdots$ \\
\hline J182851.30+010908.6 & $\mathrm{N}$ & $8.77 \pm 1.72$ & $14 \pm 24$ & $\ldots$ & $\cdots$ & $\cdots$ \\
\hline $\mathrm{J} 182851.48+010947.3$ & $\mathrm{Y}$ & $0.16 \pm 0.03$ & $50 \pm 17$ & $\cdots$ & $\cdots$ & $\cdots$ \\
\hline $\mathrm{J} 182854.44+011859.7$ & $\mathrm{Y}$ & $0.18 \pm 0.02$ & $6 \pm 22$ & $0.12 \pm 0.03$ & $25 \pm 31$ & $-0.9 \pm 0.3$ \\
\hline $\mathrm{J} 182854.46+011823.7$ & $\mathrm{Y}$ & $3.13 \pm 0.32$ & $44 \pm 9$ & $5.72 \pm 1.18$ & $17 \pm 24$ & $1.2 \pm 0.1$ \\
\hline $\mathrm{J} 182854.87+011753.0$ & $\mathrm{Y}$ & $0.08 \pm 0.02$ & $>44 \pm 19$ & $0.10 \pm 0.03$ & $-\mathrm{A}-$ & $0.4 \pm 0.5$ \\
\hline $\mathrm{J} 182901.40+010434.7$ & $\mathrm{~N}$ & $0.45 \pm 0.08$ & $26 \pm 19$ & $\cdots$ & $\cdots$ & $\cdots$ \\
\hline $\mathrm{J} 182903.06+012331.0$ & $\mathrm{Y}$ & $0.36 \pm 0.07$ & $25 \pm 21$ & $\cdots$ & $\cdots$ & $\cdots$ \\
\hline $\mathrm{J} 182903.13+010346.0$ & $\mathrm{Y}$ & $0.06 \pm 0.02$ & $-\mathrm{A}-$ & $\cdots$ & $\cdots$ & $\cdots$ \\
\hline $\mathrm{J} 182905.07+012309.0$ & $\mathrm{~N}$ & $0.12 \pm 0.02$ & $38 \pm 20$ & $\cdots$ & $\cdots$ & $\cdots$ \\
\hline J182906.84+011742.7 & $\mathrm{N}$ & $6.46 \pm 0.39$ & $8 \pm 8$ & $6.10 \pm 0.53$ & $14 \pm 11$ & $-0.1 \pm 0.1$ \\
\hline $\mathrm{J} 182907.07+011801.9$ & $\mathrm{Y}$ & $2.82 \pm 0.18$ & Extended & $1.67 \pm 0.16$ & Extended & $-1.1 \pm 0.2$ \\
\hline $\mathrm{J} 182907.62+012125.1$ & $\mathrm{~N}$ & $0.10 \pm 0.02$ & $29 \pm 22$ & $<0.04$ & $\cdots$ & $<-1.9 \pm 0.3$ \\
\hline $\mathrm{J} 182910.17+012559.5$ & $\mathrm{~N}$ & $1.41 \pm 0.25$ & $71 \pm 8$ & $\cdots$ & $\cdots$ & $\cdots$ \\
\hline J182911.94+012119.4 & $\mathrm{Y}$ & $0.35 \pm 0.05$ & $28 \pm 16$ & $0.41 \pm 0.11$ & $18 \pm 31$ & $0.3 \pm 0.2$ \\
\hline $\mathrm{J} 182912.01+011415.1$ & $\mathrm{Y}$ & $0.08 \pm 0.01$ & $>14 \pm 21$ & $0.07 \pm 0.02$ & $>45 \pm 25$ & $-0.2 \pm 0.5$ \\
\hline J182913.17+010906.4 & $\mathrm{N}$ & $0.51 \pm 0.04$ & $19 \pm 12$ & $0.60 \pm 0.08$ & $8 \pm 18$ & $0.3 \pm 0.2$ \\
\hline $\mathrm{J} 182913.36+011544.3$ & $\mathrm{Y}$ & $0.20 \pm 0.03$ & $19 \pm 20$ & $0.07 \pm 0.02$ & $>40 \pm 25$ & $-2.1 \pm 0.5$ \\
\hline $\mathrm{J} 182913.79+010738.6$ & $\mathrm{~N}$ & $0.06 \pm 0.01$ & $36 \pm 25$ & $0.10 \pm 0.02$ & $>26 \pm 20$ & $0.9 \pm 0.5$ \\
\hline $\mathrm{J} 182916.11+010437.5$ & $\mathrm{~N}$ & $0.21 \pm 0.03$ & $41 \pm 16$ & $0.25 \pm 0.06$ & $52 \pm 19$ & $0.4 \pm 0.2$ \\
\hline $\mathrm{J} 182918.23+011757.7$ & $\mathrm{~N}$ & $0.38 \pm 0.05$ & $32 \pm 15$ & $0.26 \pm 0.07$ & $41 \pm 24$ & $-0.8 \pm 0.2$ \\
\hline $\mathrm{J} 182926.71+012342.1$ & $\mathrm{~N}$ & $0.18 \pm 0.02$ & $30 \pm 13$ & $0.11 \pm 0.02$ & $34 \pm 25$ & $-1.0 \pm 0.4$ \\
\hline $\mathrm{J} 182928.02+011156.5$ & $\mathrm{~N}$ & $0.19 \pm 0.02$ & $18 \pm 18$ & $0.09 \pm 0.02$ & $-\mathrm{A}-$ & $-1.5 \pm 0.5$ \\
\hline $\mathrm{J} 182928.28+011205.7$ & $\mathrm{~N}$ & $0.21 \pm 0.04$ & $52 \pm 19$ & $<0.05$ & $\cdots$ & $<-3.1 \pm 0.4$ \\
\hline $\mathrm{J} 182929.78+012158.1$ & $\mathrm{~N}$ & $0.07 \pm 0.01$ & $-\mathrm{A}-$ & $0.06 \pm 0.02$ & $-\mathrm{A}-$ & $-0.2 \pm 0.6$ \\
\hline $\mathrm{J} 182930.71+010048.3$ & $\mathrm{~N}$ & $32.10 \pm 5.60$ & Extended & $\ldots$ & $\cdots$ & $\cdots$ \\
\hline $\mathrm{J} 182932.21+012104.6$ & $\mathrm{Y}$ & $0.10 \pm 0.01$ & $52 \pm 15$ & $0.06 \pm 0.02$ & $>20 \pm 27$ & $-0.8 \pm 0.5$ \\
\hline $\mathrm{J} 182933.07+011716.3$ & $\mathrm{~N}$ & $0.27 \pm 0.03$ & $73 \pm 5$ & $0.32 \pm 0.05$ & $74 \pm 9$ & $0.3 \pm 0.2$ \\
\hline $\mathrm{J} 182934.12+010810.9$ & $\mathrm{Y}$ & $2.06 \pm 0.17$ & $16 \pm 11$ & $2.14 \pm 0.33$ & $20 \pm 18$ & $0.1 \pm 0.1$ \\
\hline $\mathrm{J} 182934.32+011513.9$ & $\mathrm{~N}$ & $0.73 \pm 0.13$ & Extended & $0.12 \pm 0.02$ & $>48 \pm 12$ & $-3.7 \pm 0.5$ \\
\hline $\mathrm{J} 182935.02+011503.2$ & $\mathrm{~N}$ & $0.35 \pm 0.05$ & $21 \pm 15$ & $0.21 \pm 0.03$ & $34 \pm 18$ & $-1.0 \pm 0.3$ \\
\hline $\mathrm{J} 182935.11+011503.6$ & $\mathrm{~N}$ & $0.50 \pm 0.07$ & Extended & $<0.05$ & $\cdots$ & $<-4.8 \pm 0.3$ \\
\hline $\mathrm{J} 182936.50+012317.0$ & $\mathrm{~N}$ & $0.29 \pm 0.04$ & $35 \pm 14$ & $0.21 \pm 0.05$ & $39 \pm 24$ & $-0.7 \pm 0.2$ \\
\hline J182937.76+010314.6 & $\mathrm{N}$ & $0.60 \pm 0.06$ & $13 \pm 16$ & $0.33 \pm 0.06$ & $32 \pm 19$ & $-1.2 \pm 0.2$ \\
\hline $\mathrm{J} 182938.87+011850.4$ & $\mathrm{Y}$ & $0.15 \pm 0.02$ & $30 \pm 16$ & $0.13 \pm 0.02$ & $35 \pm 23$ & $-0.4 \pm 0.3$ \\
\hline $\mathrm{J} 182939.09+011233.6$ & $\mathrm{Y}$ & $0.13 \pm 0.02$ & $9 \pm 24$ & $0.05 \pm 0.02$ & $>19 \pm 33$ & $-1.9 \pm 0.6$ \\
\hline $\mathrm{J} 182940.03+011051.2$ & $\mathrm{Y}$ & $0.09 \pm 0.01$ & $>32 \pm 15$ & $0.09 \pm 0.02$ & $45 \pm 23$ & $0.0 \pm 0.4$ \\
\hline $\mathrm{J} 182944.07+011921.1$ & $\mathrm{~N}$ & $4.49 \pm 0.24$ & $21 \pm 6$ & $4.64 \pm 0.27$ & $5 \pm 8$ & $0.1 \pm 0.1$ \\
\hline $\mathrm{J} 182948.83+010647.4$ & $\mathrm{~N}$ & $0.50 \pm 0.05$ & $29 \pm 13$ & $0.76 \pm 0.13$ & $6 \pm 23$ & $0.8 \pm 0.2$ \\
\hline J182948.92+011523.8* & $\mathrm{Y}$ & $0.06 \pm 0.01$ & $>62 \pm 10$ & $<0.04$ & $\cdots$ & $<-0.5 \pm 0.5$ \\
\hline $\mathrm{J} 182949.42+011526.2$ & $\mathrm{Y}$ & $0.72 \pm 0.09$ & Extended & $0.28 \pm 0.05$ & Extended & $-1.9 \pm 0.4$ \\
\hline $\mathrm{J} 182949.50+011955.8$ & $\mathrm{Y}$ & $2.44 \pm 0.16$ & $18 \pm 8$ & $1.63 \pm 0.16$ & $7 \pm 13$ & $-0.8 \pm 0.1$ \\
\hline $\mathrm{J} 182949.54+011523.8$ & $\mathrm{Y}$ & $0.16 \pm 0.02$ & $76 \pm 7$ & $<0.04$ & $\cdots$ & $<-2.5 \pm 0.3$ \\
\hline $\mathrm{J} 182949.60+011522.9$ & $\mathrm{Y}$ & $0.18 \pm 0.02$ & $71 \pm 8$ & $0.14 \pm 0.03$ & $64 \pm 14$ & $-0.5 \pm 0.4$ \\
\hline $\mathrm{J} 182949.79+011520.4$ & $\mathrm{~N}$ & $0.88 \pm 0.07$ & $43 \pm 9$ & $0.99 \pm 0.10$ & $23 \pm 13$ & $0.2 \pm 0.2$ \\
\hline $\mathrm{J} 182950.34+011515.3$ & $\mathrm{Y}$ & $0.33 \pm 0.04$ & $11 \pm 20$ & $0.20 \pm 0.04$ & $>61 \pm 18$ & $-1.0 \pm 0.4$ \\
\hline $\mathrm{J} 182951.04+011533.8$ & $\mathrm{~N}$ & $0.61 \pm 0.05$ & $41 \pm 9$ & $0.58 \pm 0.07$ & $46 \pm 11$ & $-0.1 \pm 0.2$ \\
\hline $\mathrm{J} 182951.17+011640.4$ & $\mathrm{~N}$ & $0.09 \pm 0.02$ & $>51 \pm 16$ & $0.07 \pm 0.02$ & $>30 \pm 22$ & $-0.3 \pm 0.5$ \\
\hline $\mathrm{J} 182951.17+010529.7$ & $\mathrm{Y}$ & $0.09 \pm 0.01$ & $-\mathrm{A}-$ & $<0.04$ & $\cdots$ & $<-1.7 \pm 0.3$ \\
\hline $\mathrm{J} 182951.22+012132.0$ & $\mathrm{~N}$ & $3.80 \pm 0.22$ & $20 \pm 7$ & $2.09 \pm 0.17$ & $6 \pm 11$ & $-1.2 \pm 0.2$ \\
\hline $\mathrm{J} 182951.26+012130.3$ & $\mathrm{Y}$ & $2.46 \pm 0.16$ & $26 \pm 8$ & $1.56 \pm 0.13$ & $9 \pm 12$ & $-0.9 \pm 0.2$ \\
\hline $\mathrm{J} 182952.22+011547.4$ & $\mathrm{~N}$ & $0.12 \pm 0.02$ & $16 \pm 23$ & $<0.05$ & $\cdots$ & $<-1.7 \pm 0.3$ \\
\hline $\mathrm{J} 182953.99+011229.5$ & $\mathrm{~N}$ & $0.08 \pm 0.01$ & $46 \pm 19$ & $<0.04$ & $\cdots$ & $<-1.4 \pm 0.3$ \\
\hline $\mathrm{J} 182954.30+012011.2$ & $\mathrm{Y}$ & $0.14 \pm 0.02$ & $>75 \pm 5$ & $<0.04$ & $\cdots$ & $<-2.3 \pm 0.3$ \\
\hline $\mathrm{J} 182954.31+010309.6$ & $\mathrm{~N}$ & $0.59 \pm 0.05$ & $17 \pm 14$ & $0.60 \pm 0.09$ & $11 \pm 20$ & $0.0 \pm 0.2$ \\
\hline $\mathrm{J} 182954.36+010350.4$ & $\mathrm{Y}$ & $0.07 \pm 0.01$ & $>22 \pm 18$ & $<0.04$ & $\cdots$ & $<-0.8 \pm 0.4$ \\
\hline $\mathrm{J} 182954.86+011129.3$ & $\mathrm{Y}$ & $0.10 \pm 0.01$ & $61 \pm 21$ & $0.07 \pm 0.02$ & $>22 \pm 26$ & $-0.7 \pm 0.5$ \\
\hline $\mathrm{J} 182955.76+010440.3$ & $\mathrm{Y}$ & $0.14 \pm 0.02$ & $14 \pm 21$ & $0.12 \pm 0.02$ & $28 \pm 26$ & $-0.4 \pm 0.3$ \\
\hline $\mathrm{J} 182956.96+011247.6$ & $\mathrm{~N}$ & $<0.04$ & $\cdots$ & $0.08 \pm 0.02$ & $>57 \pm 14$ & $>1.1 \pm 0.4$ \\
\hline $\mathrm{J} 182957.60+011300.2$ & $\mathrm{~N}$ & $0.05 \pm 0.01$ & $47 \pm 20$ & $<0.05$ & $\cdots$ & $<-0.1 \pm 0.5$ \\
\hline $\mathrm{J} 182957.85+011251.1$ & $\mathrm{~N}$ & $0.05 \pm 0.01$ & $>43 \pm 19$ & $<0.08$ & $\cdots$ & $<0.8 \pm 0.5$ \\
\hline
\end{tabular}


Table 2

(Continued)

\begin{tabular}{|c|c|c|c|c|c|c|}
\hline $\begin{array}{l}\text { GBS-VLA } \\
\text { Name/Position }\end{array}$ & $\begin{array}{c}\text { New } \\
\text { Detection? }^{\mathrm{a}}\end{array}$ & $\begin{array}{c}\text { Flux Density } \\
(4.5 \mathrm{GHz}) \\
(\mathrm{mJy})\end{array}$ & $\begin{array}{c}\text { Variability } \\
(4.5 \mathrm{GHz}) \\
(\%)\end{array}$ & $\begin{array}{c}\text { Flux Density } \\
\begin{array}{c}(7.5 \mathrm{GHz}) \\
(\mathrm{mJy})\end{array}\end{array}$ & $\begin{array}{c}\text { Variability } \\
(7.5 \mathrm{GHz}) \\
(\%)\end{array}$ & $\begin{array}{l}\text { Spectral } \\
\text { Index }\end{array}$ \\
\hline $\mathrm{J} 182957.89+011246.0$ & $\mathrm{~N}$ & $3.20 \pm 0.24$ & $65 \pm 4$ & $3.32 \pm 0.41$ & $62 \pm 8$ & $0.1 \pm 0.2$ \\
\hline $\mathrm{J} 182959.55+011158.1$ & $\mathrm{~N}$ & $0.07 \pm 0.01$ & $>48 \pm 12$ & $<0.05$ & $\ldots$ & $<-0.9 \pm 0.4$ \\
\hline $\mathrm{J} 182959.94+011311.3$ & $\mathrm{~N}$ & $0.12 \pm 0.02$ & $42 \pm 20$ & $0.10 \pm 0.02$ & $18 \pm 30$ & $-0.3 \pm 0.4$ \\
\hline $\mathrm{J} 183000.65+011340.0$ & $\mathrm{~N}$ & $0.16 \pm 0.02$ & $>82 \pm 3$ & $0.10 \pm 0.02$ & $>75 \pm 8$ & $-0.9 \pm 0.4$ \\
\hline $\mathrm{J} 183001.24+010205.4$ & $\mathrm{~N}$ & $0.27 \pm 0.04$ & Extended & $<0.06$ & $\ldots$ & $<-3.1 \pm 0.3$ \\
\hline $\mathrm{J} 183002.42+012405.6$ & $\mathrm{~N}$ & $0.25 \pm 0.05$ & Extended & $<0.04$ & $\ldots$ & $<-3.7 \pm 0.4$ \\
\hline $\mathrm{J} 183002.67+012258.1$ & $\mathrm{Y}$ & $0.15 \pm 0.02$ & $25 \pm 18$ & $0.16 \pm 0.04$ & $14 \pm 31$ & $0.1 \pm 0.3$ \\
\hline $\mathrm{J} 183004.14+011239.7$ & $\mathrm{Y}$ & $0.11 \pm 0.01$ & $28 \pm 19$ & $0.07 \pm 0.02$ & $>18 \pm 29$ & $-0.8 \pm 0.5$ \\
\hline $\mathrm{J} 183004.62+012234.1$ & $\mathrm{~N}$ & $0.97 \pm 0.11$ & $49 \pm 10$ & $1.06 \pm 0.25$ & $19 \pm 27$ & $0.2 \pm 0.2$ \\
\hline $\mathrm{J} 183004.65+011353.7$ & $\mathrm{Y}$ & $0.07 \pm 0.01$ & $>43 \pm 25$ & $<0.04$ & $\ldots$ & $<-1.2 \pm 0.4$ \\
\hline $\mathrm{J} 183004.98+012226.9$ & $\mathrm{Y}$ & $0.30 \pm 0.05$ & Extended & $<0.04$ & $\ldots$ & $<-3.9 \pm 0.3$ \\
\hline $\mathrm{J} 183007.29+010324.8$ & $\mathrm{Y}$ & $0.12 \pm 0.02$ & $43 \pm 24$ & $0.10 \pm 0.02$ & $34 \pm 25$ & $-0.3 \pm 0.4$ \\
\hline $\mathrm{J} 183008.31+011519.1$ & $\mathrm{~N}$ & $0.13 \pm 0.02$ & $42 \pm 15$ & $0.07 \pm 0.02$ & $>6 \pm 34$ & $-1.2 \pm 0.5$ \\
\hline $\mathrm{J} 183008.69+010631.3$ & $\mathrm{~N}$ & $3.18 \pm 0.29$ & $11 \pm 12$ & $1.99 \pm 0.35$ & $5 \pm 24$ & $-0.9 \pm 0.1$ \\
\hline $\mathrm{J} 183008.77+010257.7$ & $\mathrm{Y}$ & $0.08 \pm 0.01$ & $-\mathrm{A}-$ & $0.06 \pm 0.02$ & $>30 \pm 27$ & $-0.5 \pm 0.6$ \\
\hline $\mathrm{J} 183010.31+012345.2$ & $\mathrm{~N}$ & $0.77 \pm 0.12$ & $48 \pm 12$ & $\ldots$ & $\ldots$ & $\ldots$ \\
\hline $\mathrm{J} 183010.60+010320.7$ & $\mathrm{~N}$ & $0.19 \pm 0.02$ & $22 \pm 18$ & $0.17 \pm 0.03$ & $36 \pm 22$ & $-0.3 \pm 0.3$ \\
\hline $\mathrm{J} 183012.58+011226.8$ & $\mathrm{~N}$ & $0.07 \pm 0.01$ & $>51 \pm 14$ & $<0.04$ & $\ldots$ & $<-1.2 \pm 0.4$ \\
\hline $\mathrm{J} 183014.25+010924.1$ & $\mathrm{Y}$ & $<0.03$ & $\ldots$ & $0.10 \pm 0.03$ & $>22 \pm 29$ & $>2.1 \pm 0.6$ \\
\hline $\mathrm{J} 183014.71+011629.6$ & $\mathrm{Y}$ & $0.13 \pm 0.02$ & $40 \pm 15$ & $0.10 \pm 0.02$ & $27 \pm 26$ & $-0.6 \pm 0.4$ \\
\hline $\mathrm{J} 183015.53+012203.9$ & $\mathrm{Y}$ & $0.20 \pm 0.02$ & $32 \pm 13$ & $0.18 \pm 0.03$ & $43 \pm 20$ & $-0.2 \pm 0.3$ \\
\hline $\mathrm{J} 183016.56+011304.3$ & $\mathrm{Y}$ & $0.07 \pm 0.01$ & $>36 \pm 15$ & $0.11 \pm 0.02$ & $19 \pm 36$ & $0.8 \pm 0.5$ \\
\hline $\mathrm{J} 183016.74+010856.2$ & $\mathrm{Y}$ & $0.27 \pm 0.03$ & $22 \pm 16$ & $0.21 \pm 0.05$ & $22 \pm 28$ & $-0.5 \pm 0.2$ \\
\hline $\mathrm{J} 183018.05+011819.2$ & $\mathrm{Y}$ & $<0.03$ & $\ldots$ & $0.09 \pm 0.03$ & $>43 \pm 27$ & $>2.0 \pm 0.4$ \\
\hline $\mathrm{J} 183022.13+011738.1^{*}$ & $\mathrm{Y}$ & $0.06 \pm 0.01$ & $-\mathrm{A}-$ & $0.05 \pm 0.02$ & $>32 \pm 52$ & $-0.2 \pm 0.7$ \\
\hline $\mathrm{J} 183024.87+011323.5$ & $\mathrm{~N}$ & $0.07 \pm 0.01$ & $>20 \pm 23$ & $0.08 \pm 0.02$ & $>43 \pm 20$ & $0.5 \pm 0.6$ \\
\hline $\mathrm{J} 183025.10+012304.3$ & $\mathrm{~N}$ & $0.08 \pm 0.01$ & $37 \pm 26$ & $0.10 \pm 0.02$ & $>59 \pm 20$ & $0.6 \pm 0.5$ \\
\hline $\mathrm{J} 183031.05+011257.3$ & $\mathrm{~N}$ & $0.08 \pm 0.02$ & $43 \pm 21$ & $<0.05$ & $\ldots$ & $<-1.0 \pm 0.4$ \\
\hline $\mathrm{J} 183052.19+011915.5$ & $\mathrm{~N}$ & $0.10 \pm 0.02$ & $21 \pm 30$ & $\ldots$ & $\ldots$ & $\ldots$ \\
\hline $\mathrm{J} 183059.74+012511.7$ & $\mathrm{~N}$ & $0.96 \pm 0.15$ & $31 \pm 16$ & $\cdots$ & $\ldots$ & $\ldots$ \\
\hline $\mathrm{J} 183059.83+012516.5$ & $\mathrm{Y}$ & $0.16 \pm 0.03$ & $34 \pm 20$ & $\ldots$ & $\ldots$ & $\ldots$ \\
\hline $\mathrm{J} 183059.86+012519.0$ & $\mathrm{Y}$ & $0.20 \pm 0.04$ & $18 \pm 24$ & $\ldots$ & $\ldots$ & $\ldots$ \\
\hline $\mathrm{J} 183104.32+011309.0$ & $\mathrm{Y}$ & $0.25 \pm 0.05$ & $42 \pm 17$ & $\ldots$ & $\ldots$ & $\ldots$ \\
\hline \multicolumn{7}{|c|}{ Detected Sources in the W40 Region: } \\
\hline J183044.11-020145.6 & $\mathrm{N}$ & $1.57 \pm 0.20$ & $9 \pm 16$ & $1.27 \pm 0.32$ & $15 \pm 31$ & $-0.4 \pm 0.2$ \\
\hline J183023.27-020731.4 & $\mathrm{Y}$ & $0.25 \pm 0.06$ & $31 \pm 24$ & $0.12 \pm 0.06$ & $36 \pm 48$ & $-1.5 \pm 0.4$ \\
\hline J183101.07-021136.8 & $\mathrm{Y}$ & $0.19 \pm 0.03$ & $6 \pm 20$ & $0.14 \pm 0.03$ & $10 \pm 34$ & $-0.6 \pm 0.4$ \\
\hline $\mathrm{J} 183102.25-015658.3$ & $\mathrm{Y}$ & $3.25 \pm 0.37$ & $4 \pm 15$ & $1.32 \pm 0.31$ & $0 \pm 33$ & $-1.8 \pm 0.2$ \\
\hline $\mathrm{J} 183102.41-015706.2$ & $\mathrm{Y}$ & $2.73 \pm 0.33$ & Extended & $0.96 \pm 0.22$ & $2 \pm 32$ & $-2.1 \pm 0.2$ \\
\hline J183102.94-015917.0 & $\mathrm{Y}$ & $0.21 \pm 0.03$ & $25 \pm 16$ & $0.26 \pm 0.06$ & $50 \pm 18$ & $0.4 \pm 0.3$ \\
\hline $\mathrm{J} 183105.01-020247.6$ & $\mathrm{~N}$ & $0.09 \pm 0.02$ & $>56 \pm 10$ & $0.09 \pm 0.02$ & $>41 \pm 22$ & $-0.0 \pm 0.6$ \\
\hline $\mathrm{J} 183109.41-015442.1$ & $\mathrm{Y}$ & $0.22 \pm 0.03$ & $3 \pm 24$ & $0.16 \pm 0.04$ & Extended & $-0.7 \pm 0.4$ \\
\hline $\mathrm{J} 183113.05-021012.1$ & $\mathrm{Y}$ & $4.70 \pm 0.40$ & Extended & $1.87 \pm 0.22$ & Extended & $-1.9 \pm 0.3$ \\
\hline $\mathrm{J} 183113.23-021011.1$ & $\mathrm{Y}$ & $20.78 \pm 1.22$ & Extended & $11.50 \pm 0.95$ & Extended & $-1.2 \pm 0.2$ \\
\hline J183114.31-020852.1 & $\mathrm{Y}$ & $0.13 \pm 0.02$ & $45 \pm 14$ & $0.09 \pm 0.02$ & $12 \pm 36$ & $-0.7 \pm 0.5$ \\
\hline $\mathrm{J} 183114.82-020350.1$ & $\mathrm{~N}$ & $0.36 \pm 0.04$ & $44 \pm 9$ & $0.42 \pm 0.07$ & $50 \pm 13$ & $0.3 \pm 0.3$ \\
\hline $\mathrm{J} 183115.28-020415.2$ & $\mathrm{~N}$ & $0.80 \pm 0.07$ & $5 \pm 12$ & $0.44 \pm 0.07$ & $1 \pm 22$ & $-1.2 \pm 0.2$ \\
\hline J183118.68-015455.9 & $\mathrm{Y}$ & $0.68 \pm 0.07$ & $11 \pm 14$ & $0.50 \pm 0.10$ & $2 \pm 27$ & $-0.6 \pm 0.2$ \\
\hline J183119.86-020006.1 & $\mathrm{Y}$ & $0.19 \pm 0.03$ & $19 \pm 16$ & $0.23 \pm 0.05$ & $15 \pm 25$ & $0.3 \pm 0.3$ \\
\hline $\mathrm{J} 183120.65-020943.6$ & $\mathrm{~N}$ & $0.09 \pm 0.02$ & $>44 \pm 12$ & $0.13 \pm 0.03$ & $9 \pm 33$ & $0.7 \pm 0.5$ \\
\hline $\mathrm{J} 183122.32-020619.6$ & $\mathrm{~N}$ & $0.67 \pm 0.07$ & $91 \pm 2$ & $1.13 \pm 0.22$ & $96 \pm 5$ & $1.1 \pm 0.2$ \\
\hline $\mathrm{J} 183122.81-020930.7$ & $\mathrm{~N}$ & $0.09 \pm 0.02$ & $7 \pm 35$ & $0.11 \pm 0.03$ & $>25 \pm 23$ & $0.4 \pm 0.5$ \\
\hline $\mathrm{J} 183123.62-020535.8$ & $\mathrm{~N}$ & $3.52 \pm 0.27$ & $15 \pm 9$ & $3.32 \pm 0.45$ & $16 \pm 16$ & $-0.1 \pm 0.1$ \\
\hline $\mathrm{J} 183124.56-020231.9$ & $\mathrm{~N}$ & $0.07 \pm 0.02$ & $>21 \pm 28$ & $<0.06$ & $\ldots$ & $<-0.3 \pm 0.5$ \\
\hline $\mathrm{J} 183125.77-015506.7$ & $\mathrm{Y}$ & $2.90 \pm 0.38$ & $27 \pm 13$ & $3.05 \pm 0.83$ & $5 \pm 37$ & $0.1 \pm 0.2$ \\
\hline $\mathrm{J} 183126.02-020517.0$ & $\mathrm{~N}$ & $1.00 \pm 0.07$ & $52 \pm 5$ & $0.75 \pm 0.09$ & $61 \pm 9$ & $-0.6 \pm 0.2$ \\
\hline $\mathrm{J} 183126.27-020630.7$ & $\mathrm{Y}$ & $0.25 \pm 0.06$ & $>39 \pm 10$ & $<0.11$ & $\ldots$ & $<-1.7 \pm 0.5$ \\
\hline $\mathrm{J} 183126.85-021042.4$ & $\mathrm{Y}$ & $0.22 \pm 0.03$ & $9 \pm 19$ & $0.14 \pm 0.03$ & $40 \pm 25$ & $-1.0 \pm 0.4$ \\
\hline $\mathrm{J} 183127.30-020504.5$ & $\mathrm{~N}$ & $0.51 \pm 0.05$ & $20 \pm 11$ & $0.45 \pm 0.07$ & $36 \pm 14$ & $-0.2 \pm 0.3$ \\
\hline $\mathrm{J} 183127.45-020512.0$ & $\mathrm{~N}$ & $2.71 \pm 0.19$ & $96 \pm 1$ & $3.17 \pm 0.34$ & $97 \pm 5$ & $0.3 \pm 0.2$ \\
\hline $\mathrm{J} 183127.64-020513.5$ & $\mathrm{~N}$ & $0.88 \pm 0.08$ & Extended & $0.65 \pm 0.11$ & Extended & $-0.6 \pm 0.3$ \\
\hline $\mathrm{J} 183127.65-020509.7$ & $\mathrm{~N}$ & $0.74 \pm 0.05$ & $19 \pm 9$ & $0.77 \pm 0.08$ & $13 \pm 14$ & $0.1 \pm 0.2$ \\
\hline
\end{tabular}


Table 2

(Continued)

\begin{tabular}{|c|c|c|c|c|c|c|}
\hline $\begin{array}{l}\text { GBS-VLA } \\
\text { Name/Position }\end{array}$ & $\begin{array}{c}\text { New } \\
\text { Detection? }^{\mathrm{a}}\end{array}$ & $\begin{array}{c}\text { Flux Density } \\
(4.5 \mathrm{GHz}) \\
(\mathrm{mJy})\end{array}$ & $\begin{array}{c}\text { Variability } \\
(4.5 \mathrm{GHz}) \\
(\%)\end{array}$ & $\begin{array}{c}\text { Flux Density } \\
(7.5 \mathrm{GHz}) \\
(\mathrm{mJy})\end{array}$ & $\begin{array}{c}\text { Variability } \\
(7.5 \mathrm{GHz}) \\
(\%)\end{array}$ & $\begin{array}{l}\text { Spectral } \\
\text { Index }\end{array}$ \\
\hline J183127.67-020519.7 & $\mathrm{N}$ & $3.45 \pm 0.24$ & $6 \pm 9$ & $3.49 \pm 0.40$ & $6 \pm 15$ & $0.0 \pm 0.2$ \\
\hline J183127.78-020449.5 & $\mathrm{N}$ & $0.12 \pm 0.02$ & $23 \pm 22$ & $0.11 \pm 0.02$ & $5 \pm 42$ & $-0.2 \pm 0.5$ \\
\hline J183127.78-020823.0 & $\mathrm{Y}$ & $0.33 \pm 0.03$ & $6 \pm 14$ & $0.23 \pm 0.02$ & $14 \pm 15$ & $-0.7 \pm 0.3$ \\
\hline J183127.80-020521.9 & $\mathrm{N}$ & $1.47 \pm 0.12$ & $17 \pm 9$ & $1.24 \pm 0.15$ & $3 \pm 17$ & $-0.3 \pm 0.2$ \\
\hline J183128.01-020517.9 & $\mathrm{N}$ & $0.61 \pm 0.06$ & $27 \pm 10$ & $0.50 \pm 0.07$ & $18 \pm 16$ & $-0.4 \pm 0.2$ \\
\hline J183128.65-020529.8 & $\mathrm{N}$ & $6.76 \pm 0.55$ & Extended & $4.79 \pm 0.71$ & Extended & $-0.7 \pm 0.2$ \\
\hline J183128.67-020522.2 & $\mathrm{N}$ & $0.31 \pm 0.04$ & $17 \pm 14$ & $0.25 \pm 0.04$ & $3 \pm 22$ & $-0.4 \pm 0.3$ \\
\hline J183129.41-020541.1 & $\mathrm{N}$ & $0.18 \pm 0.03$ & $2 \pm 31$ & $0.12 \pm 0.03$ & $-\mathrm{A}-$ & $-0.8 \pm 0.5$ \\
\hline J183130.54-020530.7 & $\mathrm{N}$ & $0.26 \pm 0.05$ & $18 \pm 27$ & $<0.07$ & $\ldots$ & $<-2.8 \pm 0.4$ \\
\hline J183148.61-020700.7 & $\mathrm{N}$ & $0.08 \pm 0.02$ & $>41 \pm 16$ & $<0.05$ & $\cdots$ & $<-0.7 \pm 0.4$ \\
\hline J183153.39-020959.9 & $\mathrm{N}$ & $0.13 \pm 0.02$ & $36 \pm 20$ & $0.08 \pm 0.03$ & $>6 \pm 44$ & $-0.9 \pm 0.5$ \\
\hline J183201.69-020243.2 & $\mathrm{Y}$ & $0.71 \pm 0.13$ & $4 \pm 25$ & $\cdots$ & $\cdots$ & $\cdots$ \\
\hline J183201.76-021012.1 & $\mathrm{Y}$ & $0.15 \pm 0.03$ & $21 \pm 27$ & $\cdots$ & $\cdots$ & $\cdots$ \\
\hline \multicolumn{7}{|c|}{ Detected Sources in the Serpens South cluster: } \\
\hline J182933.58-014530.4 & $\mathrm{Y}$ & $0.24 \pm 0.06$ & $>32 \pm 24$ & $\cdots$ & $\cdots$ & $\cdots$ \\
\hline J182940.34-015127.9 & $\mathrm{N}$ & $<0.08$ & $\cdots$ & $0.11 \pm 0.03$ & $-\mathrm{A}-$ & $>0.6 \pm 0.3$ \\
\hline J182952.73-015159.9 & $\mathrm{Y}$ & $0.23 \pm 0.03$ & $25 \pm 25$ & $0.21 \pm 0.04$ & $40 \pm 26$ & $-0.2 \pm 0.3$ \\
\hline J183003.72-014944.3 & $\mathrm{Y}$ & $0.12 \pm 0.03$ & $>45 \pm 19$ & $\cdots$ & $\cdots$ & $\cdots$ \\
\hline J183004.81-020227.8 & $\mathrm{N}$ & $<0.06$ & $\cdots$ & $0.08 \pm 0.02$ & $-\mathrm{A}-$ & $>0.7 \pm 0.5$ \\
\hline J183009.68-020032.7 & $\mathrm{Y}$ & $0.57 \pm 0.06$ & $43 \pm 10$ & $0.64 \pm 0.12$ & $19 \pm 23$ & $0.2 \pm 0.2$ \\
\hline J183018.69-020249.8 & $\mathrm{Y}$ & $0.20 \pm 0.04$ & $>18 \pm 19$ & $<0.09$ & $\cdots$ & $<-1.5 \pm 0.3$ \\
\hline J183025.24-021051.9 & $\mathrm{N}$ & $0.10 \pm 0.03$ & $-A-$ & $<0.10$ & $\ldots$ & $<-0.0 \pm 0.4$ \\
\hline J183031.68-020954.3 & $\mathrm{Y}$ & $0.25 \pm 0.05$ & $>47 \pm 13$ & $\cdots$ & $\cdots$ & $\cdots$ \\
\hline J183037.08-021503.3 & $\mathrm{Y}$ & $1.06 \pm 0.18$ & $7 \pm 23$ & $\cdots$ & $\cdots$ & $\cdots$ \\
\hline J183038.25-021100.4 & $\mathrm{Y}$ & $0.26 \pm 0.07$ & $>8 \pm 23$ & $\cdots$ & $\cdots$ & $\cdots$ \\
\hline
\end{tabular}

Note. Flux densities are measured in the combined epoch images. The quoted flux errors comprise the statistical error provided by IMFIT, the 5\% absolute flux uncertainty, and the uncertainty induced by the pointing error of the VLA primary beam. The asterisk indicates a source without reported counterparts detected with an integrated flux $<5 \sigma$, but with a peak flux $\geqslant 5 \sigma$. The $A$ annotation indicates a source not detected at three times the noise level on individual epochs, but detected on the image of the concatenated epochs.

${ }^{\mathrm{a}} \mathrm{Y}=$ source without reported counterparts at any frequency. $\mathrm{N}=$ source with known counterpart.

systematic uncertainty of $5 \%$ from possible errors in the absolute flux calibration, and the uncertainty induced by the pointing error of the VLA primary beam, which was included following Dzib et al. (2014). Adding the three errors in quadrature, we obtained the total flux uncertainties listed in columns 3 and 5 of Table 2 . When a source is detected only at one frequency, an upper limit on the flux density of that source at the other band is given. The upper limit corresponds to three times the rms noise of the area around the source position. We adopted the same criteria as Dzib et al. (2013) to consider a detection as firm. For new sources, i.e., those without reported counterparts in the literature, we considered $5 \sigma$ detections, where $\sigma$ is the rms noise of the area around the source. For known sources with counterparts in the literature, on the other hand, we included $4 \sigma$ detections. According to these criteria, we detected 94 sources in the Serpens molecular cloud, 41 in the W40 region, and 8 in the Serpens South cluster, for a total of 143 detections. Out of the 143 sources, 69 are new detections (see Section 3.2).

In order to compare the flux density between epochs and then to estimate the flux variability on a timescale of months, we searched in the images obtained from the individual epochs the sources detected in the combined ones. The flux densities and positions of the sources in these individual images are not shown for the sake of brevity.

We investigated the number of false positives that could appear given the considerably large size of our mosaics.
Assuming a Gaussian noise distribution, we estimated that the number of possible false sources with a $5 \sigma$ flux level is 7 at both 4.5 and $7.5 \mathrm{GHz}$ in the Serpens mosaic. In the W40 mosaic, the number of possible false sources is 4 and 9 at 4.5 and $7.5 \mathrm{GHz}$, respectively. For a $6 \sigma$ flux level, we found that the number of false sources is below 0.03 for both mosaics and at both frequencies. In Serpens, five sources were detected at $4.5 \mathrm{GHz}$ with flux levels between $5 \sigma$ and $6 \sigma$ and without counterparts at any other wavelengths (including counterparts at $7.5 \mathrm{GHz}$ ). At $7.5 \mathrm{GHz}$, only one source without counterparts (including counterparts at $4.5 \mathrm{GHz}$ ) was detected with a $5 \sigma-6 \sigma$ flux level. Therefore, these six sources could be false detections. In W40, all the sources without counterparts (including counterparts at 4.5 or $7.5 \mathrm{GHz}$ ) were detected above $6 \sigma$, and therefore all sources reported in that region are real detections. We noted that in both mosaics, we consider a radio source to have a counterpart at either 4.5 or $7.5 \mathrm{GHz}$ only if it is detected at above $6 \sigma$.

\subsection{Source Counterparts}

GBS-VLA source positions were compared with source positions from X-ray, optical, near-IR, mid-IR, and radio catalogs. GBS-VLA sources were considered to have a counterpart at another wavelength when the positional coincidences were better than the combined uncertainties of the two data sets. These were about $1^{\prime \prime}$ for the IR catalogs. For the Xray and radio catalogs it depends on the instrument and its 
Table 3

Radio Sources with Known Counterparts

\begin{tabular}{|c|c|c|c|c|c|c|c|}
\hline \multirow[b]{2}{*}{ GBS-VLA Name } & \multirow{2}{*}{$\begin{array}{l}\text { Other } \\
\text { Names }\end{array}$} & \multirow[b]{2}{*}{$\mathrm{X}$-ray ${ }^{\mathrm{a}}$} & \multicolumn{3}{|c|}{$\mathrm{IR}^{\mathrm{b}}$} & \multirow[b]{2}{*}{$\operatorname{Radio}^{\mathrm{c}}$} & \multirow{2}{*}{$\begin{array}{c}\text { Object } \\
\text { Type }\end{array}$} \\
\hline & & & $S S T$ & $2 \mathrm{M}$ & $\overline{W I S E}$ & & \\
\hline \multicolumn{8}{|c|}{$\begin{array}{l}\text { Counterparts of the Serpens Molecular } \\
\text { Cloud Sources: }\end{array}$} \\
\hline $\mathrm{J} 182851.30+010908.6$ & $\cdots$ & $\cdots$ & $\cdots$ & $\cdots$ & $\cdots$ & $\begin{array}{l}\text { NVSS } 182851 \\
\quad+010908\end{array}$ & $\cdots$ \\
\hline $\mathrm{J} 182901.40+010434.7$ & $\cdots$ & $\cdots$ & $\cdots$ & $\cdots$ & $\cdots$ & $\begin{array}{l}\text { NVSS } 182901 \\
\quad+010436\end{array}$ & $\cdots$ \\
\hline $\mathrm{J} 182905.07+012309.0$ & $\ldots$ & $\ldots$ & $\mathrm{Y}$ & $\ldots$ & $\ldots$ & $\ldots$ & $\ldots$ \\
\hline J182906.84+011742.7 & $\ldots$ & $\ldots$ & $\mathrm{Y}$ & $\ldots$ & $\ldots$ & $\ldots$ & $\ldots$ \\
\hline $\mathrm{J} 182907.62+012125.1$ & $\ldots$ & $\ldots$ & $\mathrm{Y}$ & $\ldots$ & $\ldots$ & $\ldots$ & $\ldots$ \\
\hline J182910.17+012559.5 & $\cdots$ & $\cdots$ & $\mathrm{Y}$ & $\ldots$ & $\ldots$ & $\ldots$ & $\ldots$ \\
\hline $\mathrm{J} 182913.17+010906.4$ & $\ldots$ & $\ldots$ & Y & $\ldots$ & $\ldots$ & $\ldots$ & $\ldots$ \\
\hline J182913.79+010738.6 & $\ldots$ & $\ldots$ & $\mathrm{Y}$ & $\ldots$ & $\ldots$ & $\ldots$ & $\ldots$ \\
\hline J182916.11+010437.5 & $\ldots$ & $\ldots$ & $\mathrm{Y}$ & $\ldots$ & $\ldots$ & $\ldots$ & $\ldots$ \\
\hline J182918.23+011757.7 & $\ldots$ & $\ldots$ & $\mathrm{Y}$ & $\ldots$ & $\ldots$ & $\ldots$ & $\ldots$ \\
\hline $\mathrm{J} 182926.71+012342.1$ & $\ldots$ & $\ldots$ & $\mathrm{Y}$ & $\ldots$ & $\ldots$ & $\ldots$ & $\ldots$ \\
\hline J182928.02+011156.5 & $\ldots$ & $\ldots$ & $\mathrm{Y}$ & $\ldots$ & $\ldots$ & $\ldots$ & $\ldots$ \\
\hline $\mathrm{J} 182928.28+011205.7$ & $\cdots$ & $\cdots$ & $\ldots$ & $\ldots$ & $\cdots$ & $\begin{array}{c}\text { NVSS } 182928 \\
+011203\end{array}$ & $\cdots$ \\
\hline $\mathrm{J} 182929.78+012158.1$ & $\ldots$ & GFM 6 & $\ldots$ & $\ldots$ & $\ldots$ & $\ldots$ & YSO \\
\hline $\mathrm{J} 182930.71+010048.3$ & PMN J1829+0101 & $\ldots$ & $\ldots$ & $\ldots$ & $\cdots$ & $\begin{array}{l}\text { NVSS } 182930 \\
\quad+010048\end{array}$ & $\ldots$ \\
\hline J182933.07+011716.3 & SVS76 Ser 14 & GFM 11 & $\mathrm{Y}$ & $\mathrm{Y}$ & $\mathrm{Y}$ & $\ldots$ & YSO \\
\hline $\mathrm{J} 182934.32+011513.9$ & $\ldots$ & $\ldots$ & $\mathrm{Y}$ & $\ldots$ & $\ldots$ & DCE08-210 5 & $\ldots$ \\
\hline $\mathrm{J} 182935.02+011503.2$ & DCE08-210 5 & $\ldots$ & $\mathrm{Y}$ & $\ldots$ & $\ldots$ & $\begin{array}{l}\text { NVSS } 182934 \\
\quad+011504\end{array}$ & $\cdots$ \\
\hline J182935.11+011503.6 & $\ldots$ & $\ldots$ & $\ldots$ & $\ldots$ & $\ldots$ & DCE08-210 5 & $\ldots$ \\
\hline J182936.50+012317.0 & $\ldots$ & $\ldots$ & $\mathrm{Y}$ & $\ldots$ & $\ldots$ & $\ldots$ & $\ldots$ \\
\hline $\mathrm{J} 182937.76+010314.6$ & $\cdots$ & $\cdots$ & $\ldots$ & $\ldots$ & $\cdots$ & $\begin{array}{l}\text { NVSS } 182937 \\
\quad+010316\end{array}$ & $\cdots$ \\
\hline J182944.07+011921.1 & DCE08-210 6 & $\ldots$ & $\ldots$ & $\ldots$ & $\ldots$ & $\begin{array}{l}\text { NVSS } 182944 \\
\quad+011920\end{array}$ & $\ldots$ \\
\hline $\mathrm{J} 182948.83+010647.4$ & $\ldots$ & $\ldots$ & $\mathrm{Y}$ & $\ldots$ & $\ldots$ & $\ldots$ & $\ldots$ \\
\hline $\mathrm{J} 182949.79+011520.4$ & SERPENS SMM 1 a & $\ldots$ & $\ldots$ & $\ldots$ & $\ldots$ & DCE08-210 1 & $\ldots$ \\
\hline J182951.04+011533.8 & $\ldots$ & $\ldots$ & $\ldots$ & $\ldots$ & $\ldots$ & ETC 8 & $\cdots$ \\
\hline $\mathrm{J} 182951.17+011640.4$ & V371 Ser & GFM 30 & $\mathrm{Y}$ & $\ldots$ & $\mathrm{Y}$ & ETC 9 & YSO \\
\hline $\mathrm{J} 182951.22+012132.0$ & $\ldots$ & $\ldots$ & $\ldots$ & $\ldots$ & $\cdots$ & $\begin{array}{l}\text { NVSS } 182951 \\
\quad+012131\end{array}$ & $\ldots$ \\
\hline $\mathrm{J} 182952.22+011547.4$ & Serpens SMM 10 IR & $\cdots$ & $\mathrm{Y}$ & $\cdots$ & $\mathrm{Y}$ & ETC 10 & YSO \\
\hline J182953.99+011229.5 & $\ldots$ & $\ldots$ & $\mathrm{Y}$ & $\ldots$ & $\ldots$ & $\ldots$ & $\ldots$ \\
\hline J182954.31+010309.6 & $\ldots$ & $\ldots$ & $\mathrm{Y}$ & $\ldots$ & $\ldots$ & $\ldots$ & $\ldots$ \\
\hline $\mathrm{J} 182956.96+011247.6$ & EC92 84 & GFM 44 & $\mathrm{Y}$ & $\mathrm{Y}$ & $\ldots$ & ETC 14 & YSO \\
\hline $\mathrm{J} 182957.60+011300.2$ & EES2009 Ser-emb 22 & GFM 46 & $\mathrm{Y}$ & $\mathrm{Y}$ & $\ldots$ & ETC 15 & YSO \\
\hline $\mathrm{J} 182957.85+011251.1$ & EES2009 Ser-emb 23 & GFM 53 & $\mathrm{Y}$ & $\mathrm{Y}$ & $\ldots$ & ETC 17 & YSO \\
\hline $\mathrm{J} 182957.89+011246.0$ & EC92 95 & GFM 54 & $\mathrm{Y}$ & $\mathrm{Y}$ & $\ldots$ & DCE08-215 8 & YSO \\
\hline $\mathrm{J} 182959.55+011158.1$ & EES2009 Ser-emb 24 & GFM 60 & $\mathrm{Y}$ & $\mathrm{Y}$ & $\cdots$ & $\ldots$ & YSO \\
\hline $\mathrm{J} 182959.94+011311.3$ & EES2009 Ser-emb 19 & $\ldots$ & $\mathrm{Y}$ & $\ldots$ & $\mathrm{Y}$ & $\ldots$ & YSO \\
\hline $\mathrm{J} 183000.65+011340.0$ & CK 6 & GFM 65 & $\mathrm{Y}$ & $\mathrm{Y}$ & $\mathrm{Y}$ & ETC 20 & YSO \\
\hline $\mathrm{J} 183001.24+010205.4$ & $\cdots$ & $\cdots$ & $\cdots$ & $\ldots$ & $\cdots$ & $\begin{array}{l}\text { NVSS } 183001 \\
\quad+010204\end{array}$ & $\cdots$ \\
\hline $\mathrm{J} 183002.42+012405.6$ & $\ldots$ & $\ldots$ & $\mathrm{Y}$ & $\cdots$ & $\mathrm{Y}$ & $\cdots$ & $\cdots$ \\
\hline $\mathrm{J} 183004.62+012234.1$ & P2003 J183004.7+012232 & GFM 70 & $\ldots$ & $\ldots$ & $\ldots$ & $\ldots$ & $\ldots$ \\
\hline $\mathrm{J} 183008.31+011519.1$ & $\ldots$ & $\ldots$ & Y & $\ldots$ & $\ldots$ & $\ldots$ & $\ldots$ \\
\hline $\mathrm{J} 183008.69+010631.3$ & $\ldots$ & $\ldots$ & $\ldots$ & $\ldots$ & $\cdots$ & $\begin{array}{l}\text { NVSS } 183008 \\
\quad+010634\end{array}$ & $\cdots$ \\
\hline $\mathrm{J} 183010.31+012345.2$ & $\ldots$ & $\ldots$ & $\mathrm{Y}$ & $\ldots$ & $\ldots$ & $\ldots$ & $\ldots$ \\
\hline $\mathrm{J} 183010.60+010320.7$ & $\ldots$ & $\ldots$ & $\mathrm{Y}$ & $\ldots$ & $\ldots$ & $\ldots$ & $\ldots$ \\
\hline $\mathrm{J} 183012.58+011226.8$ & $\ldots$ & GFM 81 & $\mathrm{Y}$ & $\ldots$ & $\cdots$ & $\cdots$ & $\ldots$ \\
\hline $\mathrm{J} 183024.87+011323.5$ & HD 170634 & $\ldots$ & $\mathrm{Y}$ & $\mathrm{Y}$ & $\mathrm{Y}$ & $\ldots$ & B7V \\
\hline $\mathrm{J} 183025.10+012304.3$ & $\ldots$ & $\ldots$ & $\mathrm{Y}$ & $\ldots$ & $\ldots$ & $\ldots$ & $\ldots$ \\
\hline $\mathrm{J} 183031.05+011257.3$ & $\ldots$ & $\ldots$ & $\mathrm{Y}$ & $\ldots$ & $\ldots$ & $\ldots$ & $\ldots$ \\
\hline $\mathrm{J} 183052.19+011915.5$ & $\ldots$ & $\ldots$ & $\mathrm{Y}$ & $\ldots$ & $\ldots$ & $\ldots$ & $\ldots$ \\
\hline J183059.74+012511.7 & $\cdots$ & $\cdots$ & $\ldots$ & $\cdots$ & $\cdots$ & $\begin{array}{l}\text { NVSS } 183059 \\
\quad+012512\end{array}$ & $\cdots$ \\
\hline
\end{tabular}


Table 3

(Continued)

\begin{tabular}{|c|c|c|c|c|c|c|c|}
\hline \multirow[b]{2}{*}{ GBS-VLA Name } & \multirow{2}{*}{$\begin{array}{l}\text { Other } \\
\text { Names }\end{array}$} & \multirow[b]{2}{*}{$\mathrm{X}$-ray ${ }^{\mathrm{a}}$} & \multicolumn{3}{|c|}{$\mathrm{IR}^{\mathrm{b}}$} & \multirow[b]{2}{*}{$\operatorname{Radio}^{c}$} & \multirow{2}{*}{$\begin{array}{l}\text { Object } \\
\text { Type }\end{array}$} \\
\hline & & & $\overline{S S T}$ & $2 \mathrm{M}$ & $\overline{W I S E}$ & & \\
\hline \multicolumn{8}{|c|}{ Counterparts of the W40 Region Sources: } \\
\hline J183044.11-020145.6 & $\cdots$ & $\cdots$ & $\cdots$ & Y & $\cdots$ & $\ldots$ & $\ldots$ \\
\hline J183105.01-020247.6 & $\begin{array}{c}\text { CXOW40 } \\
\text { J183105.02-020247.5 }\end{array}$ & KGF 18 & $\cdots$ & $\mathrm{Y}$ & $\cdots$ & $\cdots$ & YSO \\
\hline J183114.82-020350.1 & W 40 IRS 5 & KGF 36 & $\cdots$ & $\mathrm{Y}$ & $\cdots$ & RRR W40-VLA 1 & $\mathrm{~B} 1 \mathrm{~V}$ \\
\hline J183115.28-020415.2 & $\begin{array}{c}\text { CXOW40 J183115.30- } \\
020415.2\end{array}$ & KGF 38 & $\cdots$ & $\cdots$ & $\cdots$ & RRR W40-VLA 2 & $\cdots$ \\
\hline J183120.65-020943.6 & $\begin{array}{c}\text { CXOW40 J183120.65- } \\
020944.1\end{array}$ & KGF 71 & $\cdots$ & $\mathrm{Y}$ & $\cdots$ & $\cdots$ & YSO \\
\hline J183122.32-020619.6 & $\begin{array}{c}\text { CXOW40 J183122.32- } \\
020619.5\end{array}$ & KGF 82 & $\cdots$ & $\mathrm{Y}$ & $\cdots$ & RRR W40-VLA 3 & YSO \\
\hline J183122.81-020930.7 & $\begin{array}{c}\text { CXOW40 J183122.82- } \\
020930.5\end{array}$ & KGF 88 & $\cdots$ & $\mathrm{Y}$ & $\cdots$ & $\cdots$ & YSO \\
\hline J183123.62-020535.8 & $\begin{array}{c}\text { CXOW40 J183123.62- } \\
020535.7\end{array}$ & KGF 97 & $\ldots$ & $\mathrm{Y}$ & $\cdots$ & RRR W40-VLA 5 & YSO \\
\hline J183124.56-020231.9 & $\begin{array}{c}\text { CXOW40 J183124.57- } \\
020231.9\end{array}$ & KGF 102 & $\cdots$ & $\mathrm{Y}$ & $\cdots$ & $\cdots$ & YSO \\
\hline J183126.02-020517.0 & W 40 IRS $1 \mathrm{c}$ & KGF 122 & $\cdots$ & Y & $\mathrm{Y}$ & RRR W40-VLA 8 & YSO \\
\hline J183127.30-020504.5 & $\ldots$ & $\ldots$ & $\ldots$ & $\mathrm{Y}$ & $\cdots$ & RRR W40-VLA 9 & YSO? \\
\hline $\mathrm{J} 183127.45-020512.0$ & $\begin{array}{c}\text { CXOW40 J183127.46- } \\
020511.9\end{array}$ & KGF 133 & $\cdots$ & $\mathrm{Y}$ & $\cdots$ & RRR W40-VLA 10 & YSO? \\
\hline \multirow[t]{2}{*}{ J183127.64-020513.5 } & $\begin{array}{c}\text { CXOW40 J183127.64- } \\
020513.5\end{array}$ & KGF 136 & $\cdots$ & $\mathrm{Y}$ & $\cdots$ & RRR W40-VLA 12 & gyrosynchrotron \\
\hline & & & & & & & source? \\
\hline J183127.65-020509.7 & W 40 IRS $1 \mathrm{~d}$ & KGF 138 & $\cdots$ & $\mathrm{Y}$ & $\cdots$ & RRR W40-VLA 13 & YSO \\
\hline J183127.67-020519.7 & $\cdots$ & $\cdots$ & $\cdots$ & $\mathrm{Y}$ & $\cdots$ & RRR W40-VLA 14 & $\begin{array}{l}\text { gyrosynchrotron } \\
\text { source? }\end{array}$ \\
\hline J183127.78-020449.5 & $\begin{array}{c}\text { CXOW40 J183127.78- } \\
020449.5\end{array}$ & KGF 139 & $\cdots$ & $\mathrm{Y}$ & $\cdots$ & $\cdots$ & YSO \\
\hline J183127.80-020521.9 & W 40 IRS 1 a N & $\cdots$ & $\cdots$ & $\mathrm{Y}$ & $\cdots$ & RRR W40-VLA 15 & YSO \\
\hline J183128.01-020517.9 & $\begin{array}{c}\text { CXOW40 J183128.01- } \\
020517.1\end{array}$ & KGF 144 & $\cdots$ & $\mathrm{Y}$ & $\cdots$ & RRR W40-VLA 16 & YSO \\
\hline J183128.65-020529.8 & W 40 IRS $1 \mathrm{~b}$ & KGF 145 & $\cdots$ & $\mathrm{Y}$ & $\cdots$ & RRR W40-VLA 18 & YSO \\
\hline J183128.67-020522.2 & $\cdots$ & $\cdots$ & $\cdots$ & $\cdots$ & $\cdots$ & RRR W40-VLA 19 & shock front? \\
\hline J183129.41-020541.1 & $\begin{array}{c}\text { CXOW40 J183129.45- } \\
020541.2\end{array}$ & KGF 153 & $\cdots$ & $\mathrm{Y}$ & $\cdots$ & $\cdots$ & YSO \\
\hline J183130.54-020530.7 & $\begin{array}{c}\text { CXOW40 J183130.56- } \\
020530.6\end{array}$ & KGF 162 & $\cdots$ & $\mathrm{Y}$ & $\cdots$ & $\cdots$ & YSO \\
\hline J183148.61-020700.7 & $\begin{array}{c}\text { CXOW40 J183148.64- } \\
020755.5\end{array}$ & KGF 220 & $\cdots$ & $\mathrm{Y}$ & $\mathrm{Y}$ & $\cdots$ & YSO \\
\hline J183153.39-020959.9 & $\cdots$ & $\cdots$ & $\cdots$ & Y & $\mathrm{Y}$ & $\cdots$ & $\cdots$ \\
\hline \multicolumn{8}{|c|}{ Counterparts of the Serpens South Sources: } \\
\hline J182940.34-015127.9 & $\cdots$ & $\cdots$ & $\mathrm{Y}$ & $\ldots$ & $\cdots$ & $\cdots$ & YSO \\
\hline J183004.81-020227.8 & $\cdots$ & $\cdots$ & $\mathrm{Y}$ & $\cdots$ & $\cdots$ & $\cdots$ & YSO \\
\hline J183025.24-021051.9 & $\cdots$ & $\cdots$ & $\mathrm{Y}$ & $\cdots$ & $\cdots$ & $\cdots$ & YSO \\
\hline
\end{tabular}

${ }^{\text {a }}$ GFM-Giardino et al. (2007), KGF-Kuhn et al. (2010).

b SST—Evans et al. (2009), c2d-GB clouds catalog (Dunham et al. 2013), 2M-Cutri et al. (2003), and WISE —Wright et al. (2010).

${ }^{c}$ ETC-Eiroa et al. (2005); NVSS-Condon et al. (1998), DCE08-AMI Consortium et al. (2012), RRR—Rodríguez et al. (2010).

configuration. The search was done in SIMBAD and included all the major catalogs (listed explicitly in the footnote of Table 3). We have also accessed the lists with all YSOs in the c2d-GB clouds compiled by Dunham et al. (2013) and L. E. Allen et al. (2015, in preparation). In total, 354 c2d-GB sources lie inside the regions observed by us. In order to find their radio counterparts, we imaged regions of 64 pixels in each dimension, centered in the c2d-GB positions, and combining accordingly with each region, the three or two epochs. For this search we only used the field whose phase center was closest to the source. Three additional radio sources were found in Serpens South in this pursuit, increasing the number of the radio detections to 146 .

Out of 146 GBS-VLA sources, only 36 had previously been detected at radio wavelengths (column 7 of Table 3), while the other 110 are new radio detections from this survey. On the other hand, we found a total of 63 counterparts at Xray, near-IR, and mid-IR wavelengths, some of which have known radio counterparts. In total, the number of sources that were previously known (at any frequency) is 77 , while 69 of the sources in our sample are reported here for the first time. 
The classification of the sources shown in column 8 of Table 3 was taken from the literature and is based on the IR and X-ray properties of the sources. A total of 29 of the 77 sources with counterparts are firmly classified as YSOs, while HD 170634 (GBS-VLA J183024.87+011323.5) and W 40 IRS 5 (GBS-VLA J183114.82-020350.1) are typed as B7V and B1V stars, respectively. Two additional sources (GBS-VLA $\mathrm{J} 183127.30-020504.5$ and J183127.45-020512.0) are considered YSO candidates (Rodríguez et al. 2010). Three other sources have been associated with phenomena related to YSOs. Rodríguez et al. (2010) suggest that GBS-VLA J183128.67-020522.2 could correspond to a shock front from a thermal jet (possibly powered by GBS-VLA J183128.65-020529.8) interacting with the ambient interstellar medium. The sources GBS-VLA J183127.64-020513.5 and J183127.67-020519.7 have been considered as ultracompact H II ( $\left.\mathrm{UCH}_{\mathrm{II}}\right)$ region candidates, centered around young massive stars. However, Shuping et al. (2012) argue that this classification is unlikely, as the size of the unresolved radio sources at a distance of $500 \mathrm{pc}$ would be less than $100 \mathrm{AU}$, much smaller than a typical UCH II region (Kurtz 2005). Instead, they propose that the $3.6 \mathrm{~cm}$ continuum flux could be due to free-free emission from shocked gas within $100 \mathrm{AU}$ of the YSO caused by a jet or outflow. We note that, out of the 15 radio sources reported by Rodríguez et al. (2010) with IR counterparts, 7 were found by them not to be time variable (sources GBS-VLA J183114.82-020350.1， J183122.32020619.6, J183127.64-020513.5, J183127.67-020519.7, $\mathrm{J} 183127.80-020521.9$, J183128.01-020517.9, and J183128.65020529.8). In our new observations, only GBS-VLA $\mathrm{J} 183114.82-020350.1$ and $\mathrm{J} 183122.32-020619.6$ are found to have high flux variability. However, when comparing our $7.5 \mathrm{GHz}$ observations made in 2011 to those made at $8.3 \mathrm{GHz}$ in 2003 and 2004 by Rodríguez et al. (2010), remarkably, all sources show significant variations, typically by factors of $\sim 2$ (see the Appendix). We then conclude that the interpretation of the sources GBS-VLA J183127.64-020513.5 and J183127.67-020519.7 as steady UCH II regions (Rodríguez et al. 2010) is not correct, and that they are possibly gyrosynchrotron sources of slow time variability. If this is the case, they should be detectable as VLBI sources. The remaining 41 GBS-VLA sources with known counterparts at other wavelengths are, to our knowledge, not classified in the literature. In summary, we report a total of 110 unclassified sources, i.e., the 69 new detections plus the 41 sources that have previously been detected at other wavelengths but without a classification given in the literature.

\subsection{Spectral Index, Variability, and Circular Polarization}

An estimation of the radio spectral index $\alpha$ (the flux density $\left.S_{\nu} \propto \nu^{\alpha}\right)$ was obtained for most of the sources using $\alpha=\log \left(S_{\nu}(4.5) / S_{\nu}(7.5)\right) / \log (4.5 \mathrm{GHz} / 7.5 \mathrm{GHz}), \quad$ where $S_{\nu}(4.5)$ and $S_{\nu}(7.5)$ are the flux densities at 4.5 and $7.5 \mathrm{GHz}$, respectively, and $\nu$ is the frequency of the incoming radiation. The spectral index is given in column 7 of Table 2 .

The repeated observations allowed us to estimate the flux variation between the observed epochs at each frequency. Specifically, we determined the highest and lowest fluxes $\left(S_{\nu, \max }\right.$ and $S_{\nu, \min }$, respectively) of the three or two epochs. The level of variability was then estimated as the ratio of the difference between these values to the highest measured flux, i.e., variability $=\left(\mathrm{S}_{\nu, \max }-\mathrm{S}_{\nu, \min }\right) / \mathrm{S}_{\nu, \max }$. The resulting
Table 4

Sources Detected in Circular Polarization

\begin{tabular}{lcr}
\hline \hline \multirow{2}{*}{ GBS-VLA Name } & \multicolumn{2}{c}{ Degree of Circular Polarization } \\
\cline { 2 - 3 } & $4.5 \mathrm{GHz}(\%)$ & $7.5 \mathrm{GHz}(\%)$ \\
\hline $\mathrm{J} 183113.23-021011.1$ & $1(\mathrm{~L})$ & $1(\mathrm{R})$ \\
$\mathrm{J} 183123.62-020535.8$ & $5(\mathrm{R})$ & $8(\mathrm{R})$ \\
\hline
\end{tabular}

Note. The letters indicate left $(\mathrm{L})$ or right $(\mathrm{R})$ circular polarization.

values, expressed in percentages, are given in columns 4 and 6 of Table 2. The quoted uncertainties in the table for the variability and spectral index were obtained, using standard error propagation theory, from the errors of the flux density (see Section 3.1). We considered as statistically significant only those variations that are above $3 \sigma$, where $\sigma$ is the variability error of the source. In other words, we consider a source as variable if the normalized difference between its highest and lowest flux density is greater than zero within an error of $3 \sigma$.

Circular polarized radio emission was detected only from two sources in W40 (see Table 4). The identification of these sources was done also through a visual inspection in the Stokes $V$ images and searching around the position of the radio sources detected in Stokes $I$ emission. We required the signal-to-noise ratio in the Stokes $V$ images to be greater than 5. Since the degree of circular polarization $(|V| / I)$ of both sources is lower than $10 \%$, we cannot safely associate the radio emission to gyrosynchrotron. As a consequence of the smaller size of the Stokes $V$ images $\left(4^{\prime} \times 4^{\prime}\right)$, only 76 sources out of 143 were tested. The rest of them lie outside of the Stokes $V$ maps, but they could have circular polarization.

Given the existing deep X-ray, IR, millimeter, and submillimeter surveys carried out toward these regions, it is unlikely that a large fraction of the 110 unclassified sources are unidentified YSOs; however, we cannot rule out this possibility. In order to characterize the nature of the unclassified sources, we analyze their radio properties. We find that 15 radio sources in the Serpens molecular cloud and one source in the $\mathrm{W} 40$ region (Table 5) are compact and have high levels of variability ( $\gtrsim 50 \%$ at a $3 \sigma$ level) or a positive spectral index $(\geqslant+0.2$ within $1 \sigma)$. Extragalactic sources do not usually show high variability on such a short timescale (e.g., Hovatta et al. 2007, 2008; Lovell et al. 2008) or have a positive spectral index, while the radio emission seen in many Class III and some youngest objects is highly variable (e.g., Feigelson \& Montmerle 1985; Feigelson et al. 1998).

We propose then that these 16 unclassified sources are YSO candidates. The remaining 94 unclassified sources are then considered by us as extragalactic sources. Notice that sources with spectral indices larger that -0.1 could also be due to freefree radiation (Rodriguez et al. 1993). To firmly establish the nature of a radio continuum source, we need observations of its morphology, spectral index, polarization, and time variability (Rodríguez et al. 2012).

\section{DISCUSSION}

\subsection{The Lack of Radio Emission from Serpens South}

Approximately 120 YSOs from the c2d-GB catalog lie inside the region mapped by us in the Serpens South cluster. The detection of their radio counterparts is, surprisingly, very 
Table 5

Young Stellar Object Candidates Based Just on Their Radio Properties

\begin{tabular}{|c|c|c|c|}
\hline GBS-VLA Name & $\begin{array}{c}\text { Variability }(4.5 \mathrm{GHz}) \\
(\%)\end{array}$ & $\begin{array}{c}\text { Variability }(7.5 \mathrm{GHz}) \\
(\%)\end{array}$ & Spectral Index \\
\hline
\end{tabular}

YSO Candidates in the Serpens Molecular Cloud

\begin{tabular}{|c|c|c|c|}
\hline $\mathrm{J} 182854.46+011823.7$ & $44 \pm 9$ & $17 \pm 24$ & $1.2 \pm 0.1$ \\
\hline $\mathrm{J} 182910.17+012559.5$ & $71 \pm 8$ & $\cdots$ & $\cdots$ \\
\hline J182913.79+010738.6 & $36 \pm 25$ & $>26 \pm 20$ & $0.9 \pm 0.5$ \\
\hline $\mathrm{J} 182916.11+010437.5$ & $41 \pm 16$ & $52 \pm 19$ & $0.4 \pm 0.2$ \\
\hline $\mathrm{J} 182932.21+012104.6$ & $52 \pm 15$ & $>20 \pm 27$ & $-0.8 \pm 0.5$ \\
\hline J182948.83+010647.4 & $29 \pm 13$ & $6 \pm 23$ & $0.8 \pm 0.2$ \\
\hline $\mathrm{J} 182948.92+011523.8$ & $>62 \pm 10$ & $\ldots$ & $<-0.5 \pm 0.5$ \\
\hline J182949.54+011523.8 & $76 \pm 7$ & $\ldots$ & $<-2.5 \pm 0.3$ \\
\hline J182949.60+011522.9 & $71 \pm 8$ & $64 \pm 14$ & $-0.5 \pm 0.4$ \\
\hline $\mathrm{J} 182950.34+011515.3$ & $11 \pm 20$ & $>61 \pm 18$ & $-1.0 \pm 0.4$ \\
\hline $\mathrm{J} 182954.30+012011.2$ & $>75 \pm 5$ & $\ldots$ & $<-2.3 \pm 0.3$ \\
\hline $\mathrm{J} 183012.58+011226.8$ & $>51 \pm 14$ & $\ldots$ & $<-1.2 \pm 0.4$ \\
\hline $\mathrm{J} 183014.25+010924.1$ & $\cdots$ & $>22 \pm 29$ & $>2.1 \pm 0.6$ \\
\hline $\mathrm{J} 183016.56+011304.3$ & $>36 \pm 15$ & $19 \pm 36$ & $0.8 \pm 0.5$ \\
\hline $\mathrm{J} 183018.05+011819.2$ & $\ldots$ & $>43 \pm 27$ & $>2.0 \pm 0.4$ \\
\hline
\end{tabular}

YSO Candidates in the W40 Region

low. C2d-GB sources have only three radio counterparts in Serpens South (see Tables 2 and 3). These counterparts were detected with fluxes of $\lesssim 0.10 \mathrm{mJy}$ (or radio luminosities of $\lesssim 21 \times 10^{15} \mathrm{erg} \mathrm{s}^{-1} \mathrm{~Hz}^{-1}$, assuming a distance of $415 \mathrm{pc}$ ). Millimeter dust continuum data in combination with IR Spitzer observations have suggested that the Serpens South cluster is very young (a few times $10^{5} \mathrm{yr}$; Fernández-López et al. 2014). It is then expected that the radio emission from most of the YSOs in the cluster is dominated by thermal emission from strong winds. Using Equation (24) of Panagia \& Felli (1975) and following Rodriguez et al. (1989), we estimated that for a spherical wind with a terminal velocity $v=200 \mathrm{~km} \mathrm{~s}^{-1}$, an electron temperature $T_{e}=10^{4} \mathrm{~K}$, and a mass-loss rate of $10^{-7} M_{\odot} \mathrm{yr}^{-1}$, the radio flux density at a distance of $415 \mathrm{pc}$ is $\sim 0.34 \mathrm{mJy}$ at $4.5 \mathrm{GHz}$. If the cluster is more distant, i.e., at $700 \mathrm{pc}$, the radio flux decreases to $\sim 0.12 \mathrm{mJy}$ at $4.5 \mathrm{GHz}$, which is just equal to the sensitivity limit of our observations toward that region $(5 \sigma=0.12 \mathrm{mJy})$. It is then possible that we are not detecting the radio flux from the YSO population in Serpens South because the cluster is more distant than thought.

\subsection{Background Sources}

We see from the previous section that a considerable fraction of the radio sources detected are likely extragalactic objects (67 in the Serpens cloud, 19 in W40, and 8 in Serpens South, giving a total of 94 sources). In order to estimate the number of expected background radio sources, we follow Anglada et al. (1998), who took into account the Gaussian primary-beam response of the VLA antennas. Considering that at $4.5 \mathrm{GHz}$ the half-power width of the primary beam of the VLA is $\sim 10^{\prime}$, we find that the number of expected radio sources in each field with a flux greater than $S_{4.5}$ at $4.5 \mathrm{GHz}$ is given by

$$
N_{4.5}=1.21\left(\frac{S_{4.5}}{\mathrm{mJy}}\right)^{-0.75}
$$

The 25 pointings used to map the Serpens molecular cloud correspond to a total area of $25 \cdot \pi \cdot\left(10^{\prime} / 2\right)^{2}=1963.5$ square arcminutes at $4.5 \mathrm{GHz}$. However, because of the overlapping between them, the effective covered area was 900 square arcminutes at $4.5 \mathrm{GHz}$, which is equivalent to 11 fields.

Therefore, using Equation (1), we find that 105 background sources with a flux $\geqslant 64 \mu \mathrm{Jy}(5 \sigma)$ at $4.5 \mathrm{GHz}$ are predicted to lie within the mapped area of the Serpens molecular cloud. The same exercise gives 40 and 23 background sources expected in the W40 region and the Serpens South cluster, respectively. Hence, we have detected fewer extragalactic objects than expected by the count of Anglada et al. (1998) in the three regions, and we are statistically justified to assume that all of them are background and not associated with the region. Of course, this is subject to statistical variations.

\subsection{Radio Properties of the YSO Population}

We analyze the radio properties of the YSOs detected in our observations. A subset of the detected YSOs with radio emission have an SED classification reported in the literature (see Table 6 and references listed in its column 7). Out of the 29 YSOs, 8 are Class I or Flat, 5 Class II, and 12 Class III objects. In Figure 2 we plot the spectral index of these 25 objects as a function of evolutionary status. The mean values of each category are indicated by the large blue circles. We see that, given the large uncertainties involved, it is not possible to distinguish between different evolutionary classes based on the spectral index from flux densities at 4.5 and $7.5 \mathrm{GHz}$. We note, however, that there are two Class I stars with a very negative spectral index. This will be discussed later in Section 5. Notice also that most of the Class I YSOs we have detected have flux densities below $75 \mu \mathrm{Jy}(6 \sigma)$. For those sources, systematic errors are important, and the spectral index and variability errors are large. For example, GBS-VLA $\mathrm{J} 182957.60+011300.2$ could have a positive index $(+0.4)$ if we consider $1 \sigma$ dispersion. 
Table 6

Young Stellar Objects Detected in the Radio Observations

\begin{tabular}{|c|c|c|c|c|c|c|}
\hline GBS-VLA Name & $\begin{array}{c}\text { Spectral } \\
\text { Type }\end{array}$ & $\begin{array}{c}\text { SED } \\
\text { Classification }^{\mathrm{a}}\end{array}$ & $\begin{array}{c}\text { High }^{\mathrm{b}} \\
\text { Variability }\end{array}$ & $\alpha^{\mathrm{b}}$ & $\mathrm{X}$-ray & Reference $^{c}$ \\
\hline \multicolumn{7}{|c|}{ YSOs in the Serpens Molecular Cloud } \\
\hline J182929.78+012158.1 & $\ldots$ & Class II & $\ldots$ & $\mathrm{N}$ & $\mathrm{Y}$ & 1 \\
\hline $\mathrm{J} 182951.17+011640.4$ & $\cdots$ & Class I & Y & $\mathrm{N}$ & $\mathrm{Y}$ & 1,7 \\
\hline $\mathrm{J} 182952.22+011547.4$ & $\cdots$ & Class I & $\mathrm{N}$ & $\mathrm{N}$ & $\mathrm{N}$ & 2,7 \\
\hline J182956.96+011247.6 & M3.0 & Class II & Y & $\mathrm{P}$ & $\mathrm{Y}$ & 1,5 \\
\hline J182957.89+011246.0 & K1.0 & $\mathrm{P}-\mathrm{HAeBe}$ & Y & $\mathrm{F}$ & $\mathrm{Y}$ & 6 \\
\hline $\mathrm{J} 182959.55+011158.1$ & $\cdots$ & Class I & $\mathrm{N}$ & $\mathrm{N}$ & $\mathrm{Y}$ & 2,7 \\
\hline $\mathrm{J} 182959.94+011311.3$ & $\ldots$ & Class I & $\mathrm{N}$ & $\mathrm{N}$ & $\mathrm{N}$ & 2,7 \\
\hline $\mathrm{J} 183000.65+011340.0$ & M0.5 & Class III & $\mathrm{Y}$ & $\mathrm{N}$ & $\mathrm{Y}$ & 1,5 \\
\hline
\end{tabular}

YSOs in the W40 Region

\begin{tabular}{|c|c|c|c|c|c|c|}
\hline J183105.01-020247.6 & $\ldots$ & Class III & $\mathrm{Y}$ & $\mathrm{F}$ & $\mathrm{Y}$ & 3 \\
\hline J183120.65-020943.6 & $\ldots$ & Class III & $\mathrm{N}$ & $\mathrm{P}$ & $\mathrm{Y}$ & 3 \\
\hline J183122.32-020619.6 & $\ldots$ & Class III & $\mathrm{Y}$ & $\mathrm{P}$ & $\mathrm{Y}$ & 3 \\
\hline J183122.81-020930.7 & $\ldots$ & Class III & $\mathrm{N}$ & $\mathrm{P}$ & $\mathrm{Y}$ & 3 \\
\hline J183123.62-020535.8 & $\ldots$ & Class III & $\mathrm{N}$ & $\mathrm{F}$ & $\mathrm{Y}$ & 3 \\
\hline J183124.56-020231.9 & $\ldots$ & Class III & $\mathrm{N}$ & $\mathrm{N}$ & $\mathrm{Y}$ & 3 \\
\hline $\mathrm{J} 183126.02-020517.0$ & $\ldots$ & Class II & $\mathrm{Y}$ & $\mathrm{N}$ & $\mathrm{Y}$ & 4 \\
\hline $\mathrm{J} 183127.65-020509.7$ & $\ldots$ & HAeBe? ${ }^{\mathrm{d}}$ & $\mathrm{N}$ & $\mathrm{F}$ & $\mathrm{Y}$ & 3 \\
\hline J183127.78-020449.5 & $\ldots$ & Class III & $\mathrm{N}$ & $\mathrm{F}$ & $\mathrm{Y}$ & 3 \\
\hline J183127.80-020521.9 & $\ldots$ & HAeBe & $\mathrm{N}$ & $\mathrm{N}$ & $\mathrm{N}$ & 4 \\
\hline $\mathrm{J} 183128.01-020517.9$ & $\ldots$ & $\ldots$ & $\mathrm{N}$ & $\mathrm{N}$ & $\mathrm{Y}$ & 3 \\
\hline $\mathrm{J} 183128.65-020529.8$ & $\ldots$ & Class II & Extended & $\mathrm{N}$ & $\mathrm{Y}$ & 4 \\
\hline J183129.41-020541.1 & $\ldots$ & Class III & $\mathrm{N}$ & $\mathrm{N}$ & $\mathrm{Y}$ & 3 \\
\hline $\mathrm{J} 183130.54-020530.7$ & $\ldots$ & Class III & $\mathrm{N}$ & $\mathrm{N}$ & $\mathrm{Y}$ & 3 \\
\hline $\mathrm{J} 183148.61-020700.7$ & $\ldots$ & Class III & $\mathrm{N}$ & $\mathrm{N}$ & $\mathrm{Y}$ & 3 \\
\hline
\end{tabular}

\begin{tabular}{llclll}
\hline & \multicolumn{1}{c}{ YSOs in Serpens South } \\
\hline $\mathrm{J} 182940.34-015127.9$ & $\ldots$ & Flat & $\ldots$ & $\mathrm{P}$ & $\mathrm{N}$ \\
$\mathrm{J} 183004.81-020227.8$ & $\ldots$ & Class I & $\ldots$ & $\mathrm{P}$ & 7 \\
$\mathrm{~J} 183025.24-021051.9$ & $\ldots$ & Class II & $\ldots$ & $\mathrm{F}$ & $\mathrm{N}$ \\
\hline
\end{tabular}

${ }^{\mathrm{a}}$ This classification was taken from the literature; references are given in column 7.

${ }^{\mathrm{b}}$ High variability $=\mathrm{Y}$ when the flux variability is $\gtrsim 50 \%$ at a $3 \sigma$ level in at least one frequency; $\mathrm{N}$ when the variability is $<50 \%$ at both frequencies. $\alpha$ refers to the spectral index and is given as $\mathrm{P}$ (for positive) when it is higher than $0.2 ; \mathrm{F}$ (for flat) when it is between -0.2 and +0.2 ; and $\mathrm{N}$ (for negative) when it is lower than -0.2 . $\mathrm{X}$-ray $=\mathrm{Y}$ when there is an $\mathrm{X}$-ray flux reported in literature, $\mathrm{N}$ when it is not.

c (1) Giardino et al. (2007), (2) Enoch et al. (2009), (3) Kuhn et al. (2010), (4) Shuping et al. (2012), (5) Winston et al. (2010), (6) Preibisch (1999), (7) = c2d-GB cloud catalog (Dunham et al. 2013).

${ }^{\mathrm{d}}$ This source has a mass of $\sim 4 M_{\odot}$ and no $K_{s}$-band excess. We therefore consider it as an HAeBe candidate.

Out of the 25 detected Class I-III objects, 20 have a variability determination at either 4.5 or $7.5 \mathrm{GHz}$. Figure 3 shows the level of variability as a function of the evolutionary status for these 20 objects. We see that the older class (Class III) is populated by objects with very high variable emission. In the same plot, we show the weighted average of variability for each evolutionary class. While Class I and Class II sources have weighted average variability lower than $50 \%$, the weighted average variability of Class III is $\sim 80 \%$. In order to test if variability increases with age, as is suggested by the plot, we carried out a Kolmogorov-Smirnov test on the three YSO classes. The null hypothesis to test is that the variability distributions of different classes are drawn from the same distribution. Figure 4 shows the cumulative probability distributions. The $D$ statistic gives the absolute maximum distance between the cumulative distributions of two samples. The $D$ statistic is $D_{\mathrm{I}-\mathrm{II}}=1.16$ for class I and II distributions,
$D_{\mathrm{I}-\mathrm{III}}=0.89$ for class $\mathrm{I}$ and III distributions, and $D_{\text {II-III }}=0.65$ for class II and III distributions. The $p$-value gives the probability of obtaining the observed distributions when the null hypothesis is true. We obtained a $p$-value of $p_{\text {I-II }}=0.13$ for class I and II distributions, $p_{\text {I-III }}=0.4$ for class I and III distributions, and $p_{\text {II-III }}=0.79$ for class II and III distributions. Assuming that sample pairs with $p<0.10$ are taken from different distribution functions with high significance, we cannot reject the null hypothesis. This result may be affected by the small number of YSOs in the samples and the large uncertainties of the variability. However, we are interested in testing this tendency in a forthcoming paper, using the whole sample of YSOs detected toward the Ophiuchus, Orion, Perseus, and Taurus-Auriga regions.

We weighted average the variability of the YSO candidates identified in our observations (Table 5). This average is shown 


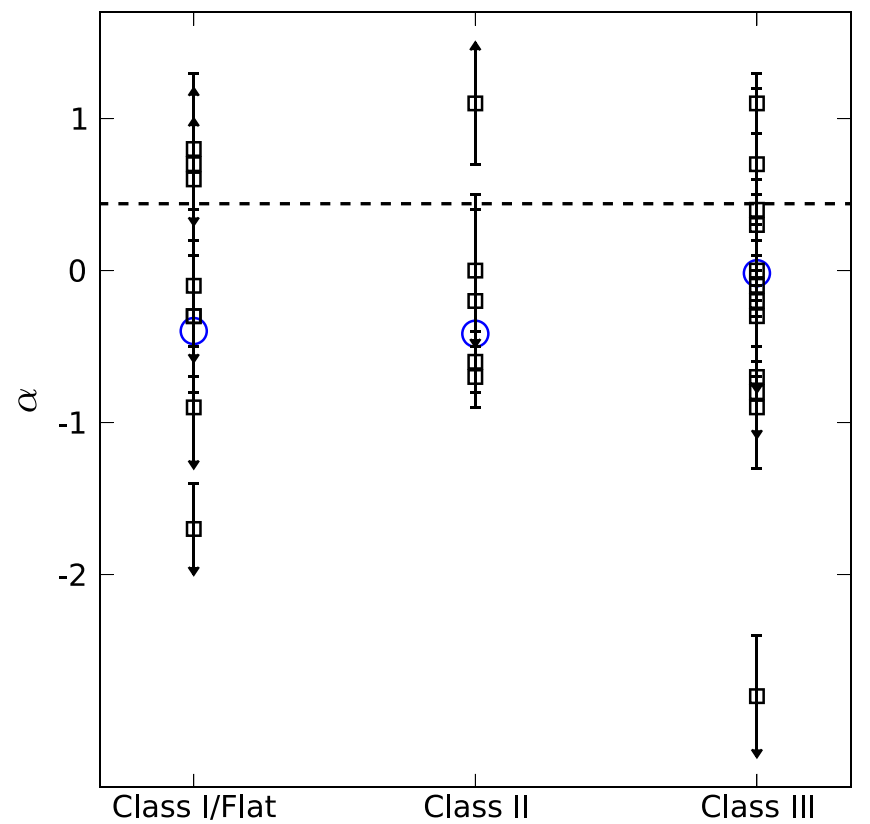

Figure 2. Spectral index as a function of evolutionary status of the 25 detected Class I-III YSOs. Values for the individual sources are shown with squares, and weighted mean values with circles. The arrows indicate upper and lower limits. The horizontal dashed line marks the weighted average spectral index of the YSO candidates listed in Table 5.

as a horizontal line in Figure 3. Interestingly, we find that the weighted average variability $(\sim 90 \%)$ of the YSO candidates is closest to the averaged variability of the Class III known YSOs. This suggests that it might be the largest population of Class III sources out of our YSO candidates.

\subsection{The X-Ray-Radio Relation}

For active stellar coronae, Güdel \& Benz (1993) found a correlation between X-ray and radio luminosities, $L_{X}$ and $L_{R}$, respectively, which holds for $X$-ray luminosities over six orders of magnitude. The interpretation of this empirical relation is that the mechanism responsible for accelerating the nonthermal electrons that emit in the radio continuum also heats the coronal plasma, and this gives rise to the thermal X-ray flux. Class Me dwarfs, Ke dwarfs, and BY Dra stars, which typically have low luminosities, satisfy

$$
\frac{L_{X}}{L_{R}} \approx 10^{15.5 \pm 1}[\mathrm{~Hz}] .
$$

More luminous classes (WTTS, RS CVn's binaries, Algols, and FK Com stars) are systematically less X-ray bright compared to their radio luminosity and fulfill

$$
L_{X} / L_{R} \lesssim 10^{15.5}[\mathrm{~Hz}]
$$

We study the $L_{X}-L_{R}$ relation for the YSOs with X-ray counterparts in our sample. A total of 29 radio sources have $\mathrm{X}$ ray counterparts, 23 of which are YSOs. However, we consider only YSOs with high radio variability or with a negative or flat spectral index (a subset of 18 Class I-III sources), thereby excluding sources that could be not coronal. Also, we have corrected all X-ray luminosities to the distance of $415 \mathrm{pc}$ adopted in this work (see the discussion in Section 1). The corrected luminosity $L_{0}$ was obtained using $L_{0}=\left(d_{0} / d\right)^{2} L$,

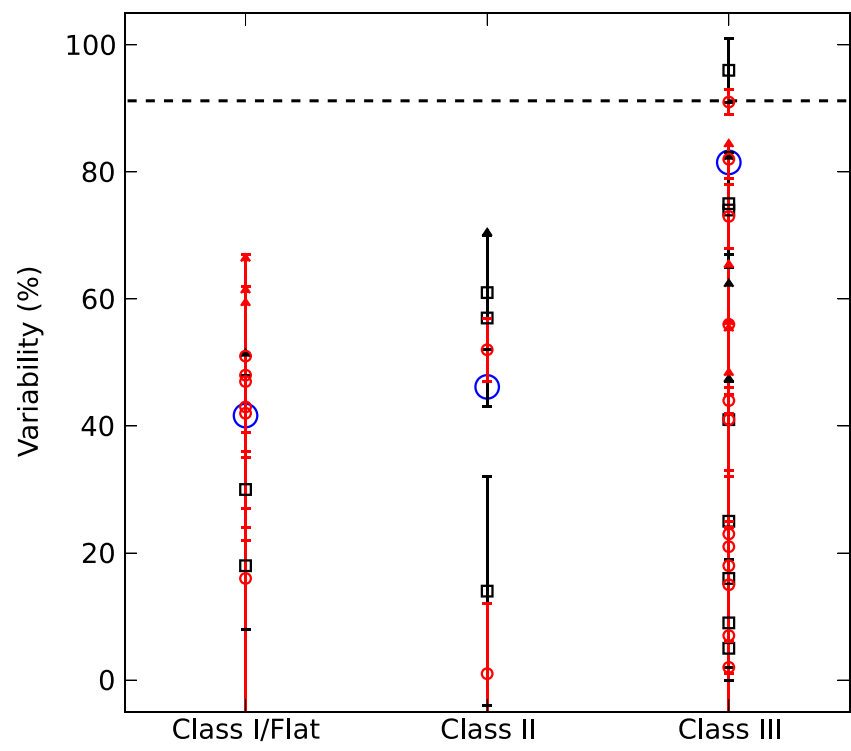

Figure 3. Variability as a function of the evolutionary status of 20 Class I-III YSOs. Values for the individual sources are shown with red circles (variability at $4.5 \mathrm{GHz}$ ) and black squares (variability at $7.5 \mathrm{GHz}$ ). Some sources have a variability determination at both frequencies. The blue circles are the weighted average for each class. The arrows indicate lower limits. The horizontal line marks the weighted average variability at both frequencies of the YSO candidates listed in Table 5.

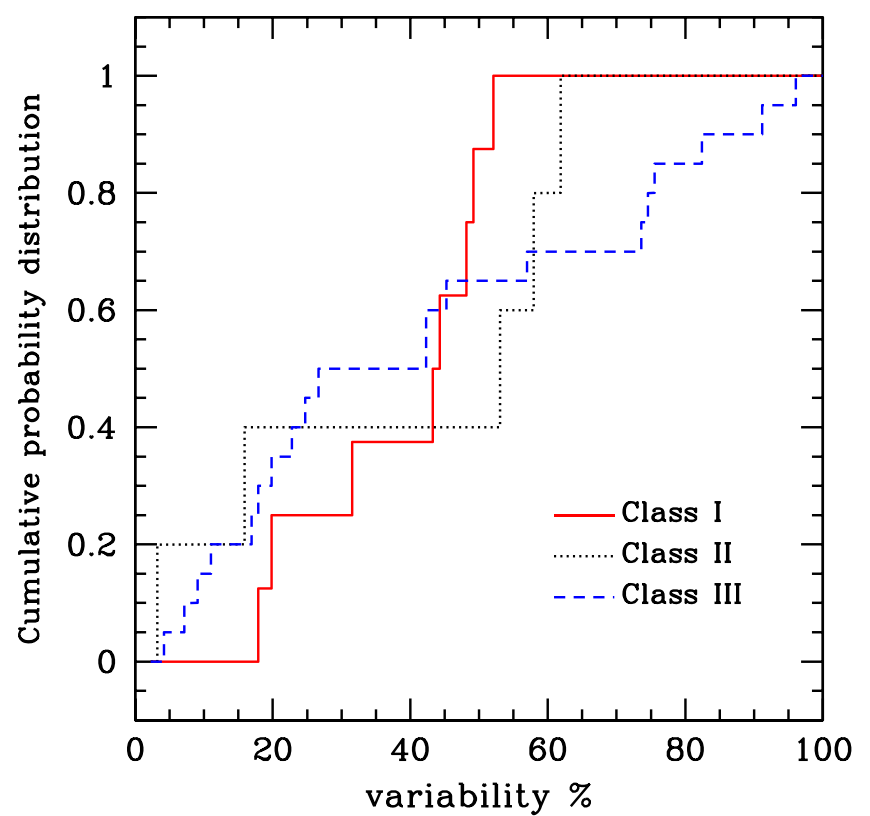

Figure 4. K-S test: cumulative probability distributions of variability in the three classes of YSOs. Red solid line: Class I; black dotted line: Class II; blue dashed line: Class III.

where $L$ is the luminosity of the source assuming a distance $d$, and $d_{0}$ is the new adopted distance.

Following Güdel \& Benz (1993), we place our subset of YSOs in the $L_{X}-L_{R}$ diagram (Figure 5) and compare with the relations already determined for stars of different classes with magnetic activity. Also plotted in Figure 5 are the YSOs detected in the Ophiuchus complex by Dzib et al. (2013). We find that the YSOs we have detected in Serpens and W40, as 


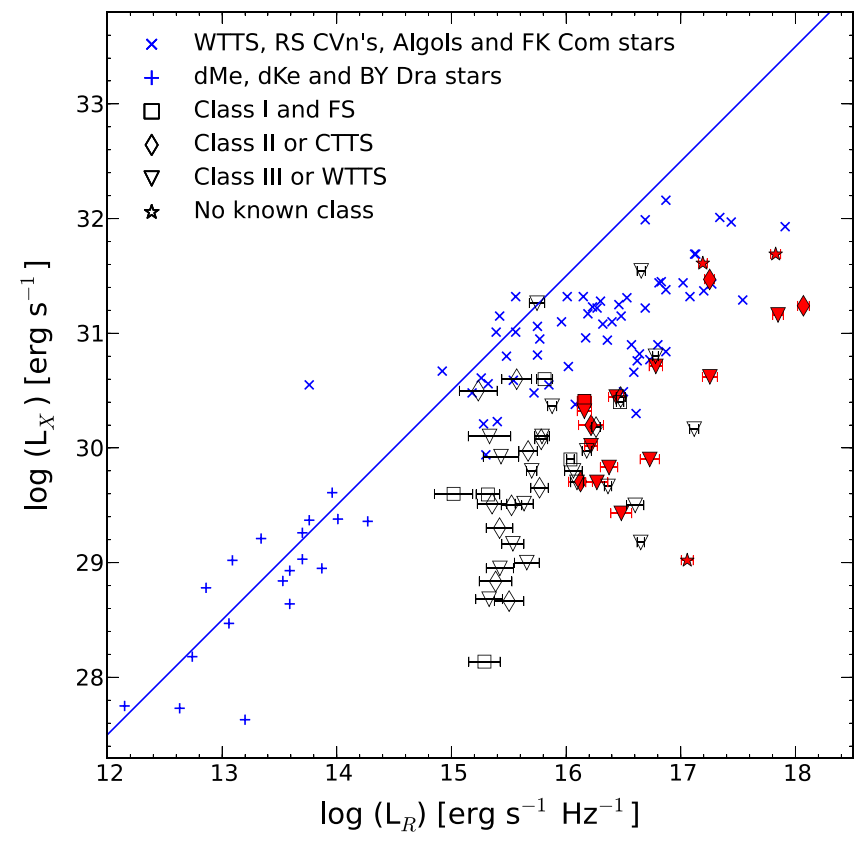

Figure 5. $L_{X}-L_{R}$ relation for stars following Güdel \& Benz (1993). Symbols indicate different classes of stars as explained at the top left of the diagram. Radio and X-ray luminosities of active stellar coronae represented by blue vertical and diagonal crosses were taken from Güdel et al. (1993) and Drake et al. (1989). The solid line with slope 1 is the fit obtained by Güdel \& Benz (1993) for the $\mathrm{dM}(\mathrm{e}), \mathrm{dK}(\mathrm{e})$, and BY Dra stars (blue vertical crosses), which occupy the lower left portion of the diagram. Open symbols correspond to YSOs detected in the Ophiuchus complex (Dzib et al. 2013). The YSOs detected by us in the Serpens and W40 regions with X-ray counterparts and with possible coronal radio emission are shown as red filled symbols. The radio luminosity used for GBS-VLA sources is the average of the luminosities at 4.5 and $7.5 \mathrm{GHz}$.

well as the sample of YSOs in Ophiuchus, do not follow the Güdel-Benz relation for dwarf stars (Equation (2)). Conversely, they fulfill $L_{X} / L_{R} \lesssim 10^{15.5}[\mathrm{~Hz}$. Güdel \& Benz (1993) proposed that the deviation from the relation with slope equal to 1 is likely a result of the sources having larger magnetospheres, which causes longer trapping times for the radio-emitting high-energy particles.

Lower X-ray fluxes than the relation given by Equation (2) could also be explained if the photons are absorbed by gas in front of the clouds. Kuhn et al. (2010) determined the visual absorption $A_{V}$ toward their W40 sources from a $J$ versus $J-H$ diagram. Our radio sources with counterparts in the catalog of Kuhn et al. (2010) have visual absorptions ranging from 6 to 22 mag. Using the standard conversion $N_{\mathrm{H}}=$ $(1.8 \pm 0.3) \times 10^{21} \mathrm{~cm}^{-2} \times A_{V} \quad($ Predehl \& Schmitt 1995), we find that the values of $A_{V}=6-22$ imply absorbing column densities of $N(\mathrm{H}) \sim(1-4) \times 10^{22} \mathrm{~cm}^{-2}$. In the Serpens core, Giardino et al. (2007) report column densities of $N(\mathrm{H}) \sim(0.5-7.4) \times 10^{22} \mathrm{~cm}^{-2}$ for our YSOs with radio emission. Another possibility for the deviation from relation (2) is a bias toward the brightest sources at the X-ray band in the Güdel-Benz relation. According to this interpretation, the full area below the relation could be populated with fainter coronal sources. In fact, the YSOs in Serpens and Ophiuchus lie below the stars analyzed in the original study of Güdel \& Benz (1993).

\subsection{Proper Motions of YSOs in the W4O Region}

Rodríguez et al. (2010) observed the $\mathrm{W} 40$ region at $3.6 \mathrm{~cm}$ with the VLA in its A and B array configurations. We use the observations obtained with the A configuration (2004 September 18) to estimate the angular displacement of the sources between then and our own observations $7 \mathrm{yr}$ later, in 2011. The phase calibrator $\mathrm{J} 1804+010$ was used in all observing runs. In total, only nine compact sources are detected at both epochs (see Table 7). Notice that the displacement of the sources generated by their trigonometric parallax $\left(p\left(^{\prime \prime}\right)=1 / D\right.$ [pc] $)$ is 2.4 mas for a distance of $D=415 \mathrm{pc}$, which is comparable to or even lower, by one order of magnitude, than the position errors of these sources (2-15 mas). Therefore, we do not consider the contribution from the parallax to the angular displacement of the sources. The proper motions in R.A., $\mu_{\alpha} \cos (\delta)$, and decl., $\mu_{\delta}$, are given in Table 7 and plotted in Figure 6 . We also see that, with the exception of GBS-VLA J183122.32-020619.6 (RRR W40-VLA 3), all the sources are moving in the same direction with a mean absolute value $\mu_{\text {total }}=12.7$ mas $\mathrm{yr}^{-1}$. The average proper motions in R.A. and decl. of these eight sources are $\mu_{\alpha} \cos (\delta)=-8.0 \operatorname{mas~yr}^{-1}$ and $\mu_{\delta}=-9.7$ mas $\mathrm{yr}^{-1}$. The sources GBS-VLA J183122.32-020619.6, J183123.62-020535.8, J183126.02-020517.0, and J183128.65-020529.8 (RRR W40-VLA 3, 5, 8, and 18) are associated with YSOs and, in fact, they all have non-zero proper motions. Rodríguez et al. (2010) suggested that GBSVLA J183127.30-020504.5 (RRR W40-VLA 9) is a YSO candidate, while we propose that GBS-VLA J183127.67-020519.7 (RRR W40-VLA 14) is a gyrosynchrotron source. The movements of these sources are similar to most of the stars reported in Table 7, confirming that both objects are Galactic. It has been posited by Rodríguez et al. (2010) that GBS-VLA J183128.67-020522.2 (RRR W40VLA 19) is a shock front from a thermal jet (possibly powered by GBS-VLA J183128.65-020529.8). We note that this source has not been detected at IR, optical, or X-ray wavelengths, which suggests that it is not a YSO. Its proper motion is the largest of the group, and this could be due to an intrinsic motion. The expected proper motions for objects in the direction toward $\mathrm{W} 40$ at a distance of $415 \mathrm{pc}$ are

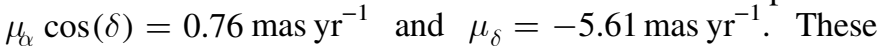
values are smaller than our estimations, and probably this could be due to a systematic error (like an offset in the position of the phase calibrator in the archive data). However, we have investigated this possibility and do not find any offset. It is also possible that the cluster has a peculiar velocity.

\section{COMMENTS ON INDIVIDUAL SOURCES}

The source W40 IRS $1 \mathrm{~d}$ (GBS-VLA J183127.65-020509.7) was classified as a single source by Smith et al. (1985), but it has been recently resolved in the near-IR into a small cluster of at least seven distinct sources by Shuping et al. (2012). The position of the X-ray source associated with it, however, comes from a high-resolution (0.'5) X-ray image of the Chandra telescope. The source is classified as an intermediate-mass YSO ( $\sim 4 M_{\odot}$; Kuhn et al. 2010) and coincident, within the error, with the position of the radio source. Thus, we will consider that W40 IRS $1 \mathrm{~d}$ is associated with this young star. Also, this source does not show $K_{s}$-band excess (Kuhn et al. 2010), a fact that, along 
Table 7

Proper Motions of Some Radio Sources in W40

\begin{tabular}{|c|c|c|c|c|}
\hline GBS-VLA Name & $\begin{array}{c}\text { RRR W40-VLA } \\
\text { Number }^{\mathrm{a}}\end{array}$ & $\begin{array}{l}\mu_{\alpha} \cos (\delta) \\
\left(\operatorname{mas} \mathrm{yr}^{-1}\right)\end{array}$ & 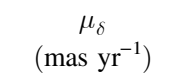 & $\begin{array}{c}\mu_{\text {total }} \\
\left(\text { mas yr }^{-1}\right)\end{array}$ \\
\hline J183122.32-020619.6 & 3 & $5.13 \pm 1.99$ & $-12.1 \pm 2.15$ & 13.14 \\
\hline J183123.62-020535.8 & 5 & $-2.43 \pm 0.4$ & $-6.83 \pm 0.42$ & 7.25 \\
\hline J183127.30-020504.5 & 9 & $-7.54 \pm 3.96$ & $-10.32 \pm 4.22$ & 12.78 \\
\hline J183127.67-020519.7 & 14 & $-9.17 \pm 0.64$ & $-7.77 \pm 0.67$ & 12.02 \\
\hline J183127.80-020521.9 & 15 & $-6.94 \pm 1.0$ & $-9.95 \pm 1.06$ & 12.13 \\
\hline J183128.67-020522.2 & 19 & $-14.97 \pm 3.8$ & $-10.38 \pm 4.14$ & 18.22 \\
\hline
\end{tabular}

a The labels in this column refer to the VLA source number in the catalog of Rodríguez et al. (2010).

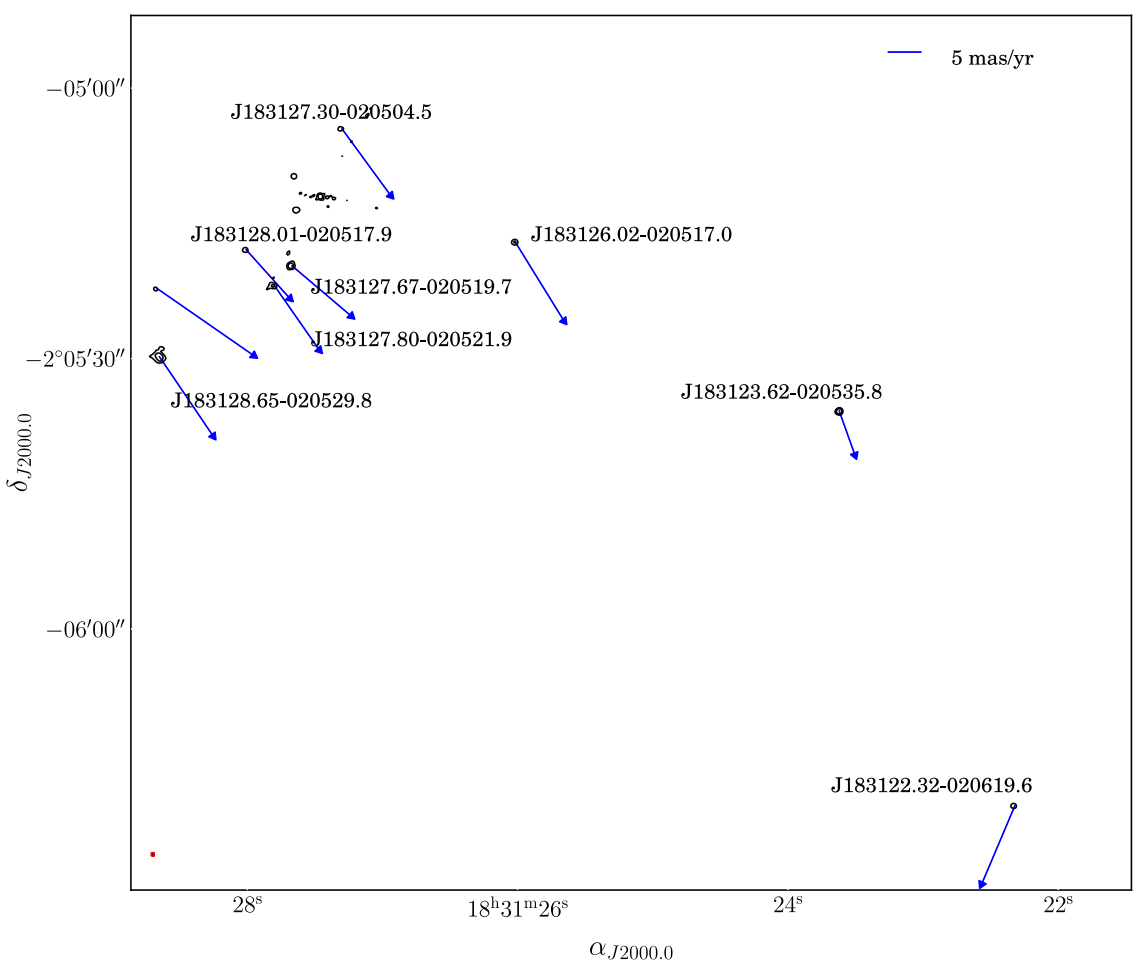

Figure $6.6 \mathrm{~cm}$ radio continuum images of the $\mathrm{W} 40$ region. The arrows indicate the direction and length of the total proper motion of radio sources in the cluster, detected by us and by Rodríguez et al. (2010). The $\theta_{\text {maj }} \times \theta_{\min }=0 . \prime 41 \times 0.40$, P.A. $=+75.8$ synthesized beam is shown in the bottom left of the map. Contours are 5 and 25 times $32 \mu \mathrm{Jy}$ beam $^{-1}$, the rms noise of the image.

with its determined mass, suggests that the source may be an HAeBe star. The source W40 IRS 5 (GBS-VLA J183114.82-020350.1) was first classified as a foreground star due to its lack of significant IR absorption (Kuhn et al. 2010). However, more recently, Shuping et al. (2012) classified this source as a B1V star. They also obtained an extinction toward it similar to that of other stars in the cluster. Moreover, the distance determined to this source $(\sim 469 \mathrm{pc})$ is coincident with that determined to three additional $\mathrm{OB}$ stars in the region. This strongly suggests that W40 IRS 5 is part of the cluster and not a foreground star, as proposed by Kuhn et al. (2010).

The sources NVSS 182934+011504 and NVSS182951 + 012131, from the catalog of Condon et al. (1998), are resolved into double sources in our observations (Figures 7 and 8), while NVSS 183059+012512 is resolved into a triple source (Figure 9). Given the angular separation between the GBS-VLA sources and the peak of their NVSS counterparts, as well as the uncertainties in their positions, we associated NVSS 182934+011504, NVSS 182951 +012131, and NVSS 183059+012512 with GBS-VLA J182935.02+011503.2， GBS-VLA J182951.22+012132.0, and GBS-VLA J183059.74+012511.7, respectively (Table 3). Three GBS-VLA sources lie inside the source DCE08-210 5 ( size $\sim 1^{\prime}$; Figure 7), detected by AMI Consortium et al. (2012), and it is likely that this source includes multiple contributions, so it is difficult to attribute the $16 \mathrm{GHz}$ emission conclusively to a specific GBS-VLA source. On the other hand, although DCE08-210 1 is resolved into six sources (Figure 10), GBS-VLA J182949.79+011520.4 is the strongest source closer to the peak of the $16 \mathrm{GHz}$ emission; therefore, we associate DCE08-210 5 with GBS-VLA $\mathrm{J} 182949.79+011520.4$. 
Table A1

W40 Sources of the Catalog of Rodríguez et al. (2010) Detected in Our Observations

\begin{tabular}{|c|c|c|c|c|c|}
\hline GBS-VLA Name & $\begin{array}{c}\text { RRR W40-VLA } \\
\text { Number }^{\mathrm{a}}\end{array}$ & $\begin{array}{c}\text { RRR Flux Density } \\
(8.3 \mathrm{GHz}) \\
(\mathrm{mJy})\end{array}$ & $\begin{array}{c}\text { GBS Flux Density } \\
(7.5 \mathrm{GHz}) \\
(\mathrm{mJy})\end{array}$ & $\begin{array}{l}\text { Spectral } \\
\text { Index }\end{array}$ & $\begin{array}{c}\text { RRR Extrapolated Flux Density } \\
(7.5 \mathrm{GHz}) \\
(\mathrm{mJy})\end{array}$ \\
\hline J183114.82-020350.1 & 1 & $0.92 \pm 0.18$ & $0.42 \pm 0.07$ & $+0.3 \pm 0.2$ & $0.89 \pm 0.18$ \\
\hline J183122.32-020619.6 & 3 & $0.47 \pm 0.09$ & $1.13 \pm 0.22$ & $+1.1 \pm 0.2$ & $0.42 \pm 0.08$ \\
\hline J183123.62-020535.8 & 5 & $3.97 \pm 0.79$ & $3.32 \pm 0.45$ & $-0.1 \pm 0.1$ & $4.01 \pm 0.80$ \\
\hline $\mathrm{J} 183126.02-020517.0$ & 8 & $3.27 \pm 0.65$ & $0.75 \pm 0.09$ & $-0.6 \pm 0.2$ & $3.48 \pm 0.70$ \\
\hline $\mathrm{J} 183127.64-020513.5$ & 12 & $1.64 \pm 0.33$ & $0.65 \pm 0.11$ & $-0.6 \pm 0.3$ & $1.74 \pm 0.35$ \\
\hline $\mathrm{J} 183127.65-020509.7$ & 13 & $0.86 \pm 0.17$ & $0.77 \pm 0.08$ & $+0.1 \pm 0.2$ & $0.85 \pm 0.17$ \\
\hline J183127.67-020519.7 & 14 & $5.78 \pm 1.16$ & $3.49 \pm 0.40$ & $+0.0 \pm 0.1$ & $5.78 \pm 1.16$ \\
\hline $\mathrm{J} 183127.80-020521.9$ & 15 & $1.71 \pm 0.34$ & $1.24 \pm 0.15$ & $-0.3 \pm 0.2$ & $1.76 \pm 0.35$ \\
\hline $\mathrm{J} 183128.01-020517.9$ & 16 & $0.94 \pm 0.19$ & $0.50 \pm 0.07$ & $-0.4 \pm 0.2$ & $0.98 \pm 0.20$ \\
\hline J183128.65-020529.8 & 18 & $11.1 \pm 2.22$ & $4.79 \pm 0.71$ & $-0.7 \pm 0.2$ & $11.9 \pm 2.38$ \\
\hline
\end{tabular}

a The numbers in this column refer to the VLA source number in the catalog of Rodríguez et al. (2010).

\subsection{Non-thermal Radio Emission from YSOs}

In Table 6 we list the radio properties of the 29 YSOs detected. As we already mentioned, non-thermal gyrosynchrotron radio emission is characterized by high radio variability and often a negative spectral index and some level of circular polarization. Out of the 12 Class III objects detected, 8 show high levels of variability or have a negative spectral index. We thus consider these eight objects as possible sources of nonthermal emission. Four Class II objects and, interestingly, four Class I sources could also be non-thermal. Additionally, the Herbig AeBe star GBS-VLA J183127.80-020521.9, the protoHerbig AeBe star (P-HAeBe) GBS-VLA J182957.89 +011246.0 , and the source GBS-VLA J183128.01-020517.9 are likely non-thermal radio sources. This gives us a high fraction $(65 \%)$ of the YSOs detected in our observations being non-thermal radio sources. An independent clue of the nature of the emission of these objects will be provided by their detection (or lack thereof) in forthcoming VLBA observations.

The Class I objects with possible non-thermal emission are GBS-VLA J182951.17+011640.4, J182952.22+011547.4, J182959.55+011158.1, and J182959.94+011311.3. Previously, some Class I objects in different star-forming regions have been reported as non-thermal emitters (Feigelson et al. 1998; Forbrich et al. 2007; Deller et al. 2013). One explanation for the detection of non-thermal radio emission from these objects (which should be absorbed by the ionized wind of the star) is a geometrical effect. According to this scenario, if the star is seen nearly pole-on or nearly edge-on, then the non-thermal radio emission originating in the corona might be less absorbed by the surrounding material and can reach the observer. Another possibility is tidal clearing of circumstellar material in a tight binary system (Dzib et al. 2010).

\subsection{EC 95}

EC $95=$ GBS-VLA J182957.89+011246.0 is a tight binary (angular separation of 15 mas) consisting of a proto-Herbig AeBe star and a possibly low-mass T Tauri companion. Both components were detected with the VLBA and are therefore non-thermal radio sources (Dzib et al. 2010). The origin of this non-thermal emission has been proposed to be intrinsic magnetic activity in the stars. The magnetic activity in the low-mass $\mathrm{T}$ Tauri companion is related to magnetic reconnection in the stellar surface. Electrons are then accelerated to mildly relativistic velocities and generate gyrosynchrotron radiation. On the other hand, intermediate-mass stars are not expected to be magnetically active. Some processes have been suggested to explain the origin of the non-thermal radio emission in the proto-Herbig AeBe star (see Dzib et al. 2010), but still need to be tested.

We derive a spectral index for the system of $0.1 \pm 0.2$, which is consistent within $1 \sigma$ with an early estimation by Smith et al. (1999), who found $-0.26 \pm 0.26$. We determined a high variability at 4.5 and $7.5 \mathrm{GHz}(65 \%$ and $62 \%$, respectively). We note that in the VLBA observations obtained by Dzib et al. (2010) both components were found to be very variable, at levels of $\sim 94 \%$, and so the variability we derive should be associated with the intrinsic variability of the stars. In conclusion, the radio properties we obtain for this system are consistent with the non-thermal nature of the sources.

\section{SUMMARY}

We have carried out new radio observations of three regions in the Aquila complex of local dark molecular clouds, namely, the Serpens molecular cloud, the Serpens South cluster, and the W40 region. We covered a large field of view $\left(\sim 0.45 \mathrm{deg}^{2}\right)$, and, combined with high angular resolution $(\sim 0.3)$ and sensitivity $(\sim 17 \mu \mathrm{Jy})$, our study surpasses previous observations of these regions. We have detected a total of 146 sources. Twenty-nine of them are associated with YSOs and 110 are new radio detections. The multi-epoch and dual-frequency strategy allows us to speculate on the nature of the radio emission of the sources. In particular, we find that 16 of the unclassified sources have a positive spectral index or exhibit high variability. They might, hence, correspond to a small population of as-yet-unidentified YSOs. What is more interesting is that approximately $65 \%$ of the identified YSOs are non-thermal sources, of which $50 \%$ are bright enough and therefore excellent targets for future astrometric observations with VLBI instruments. 


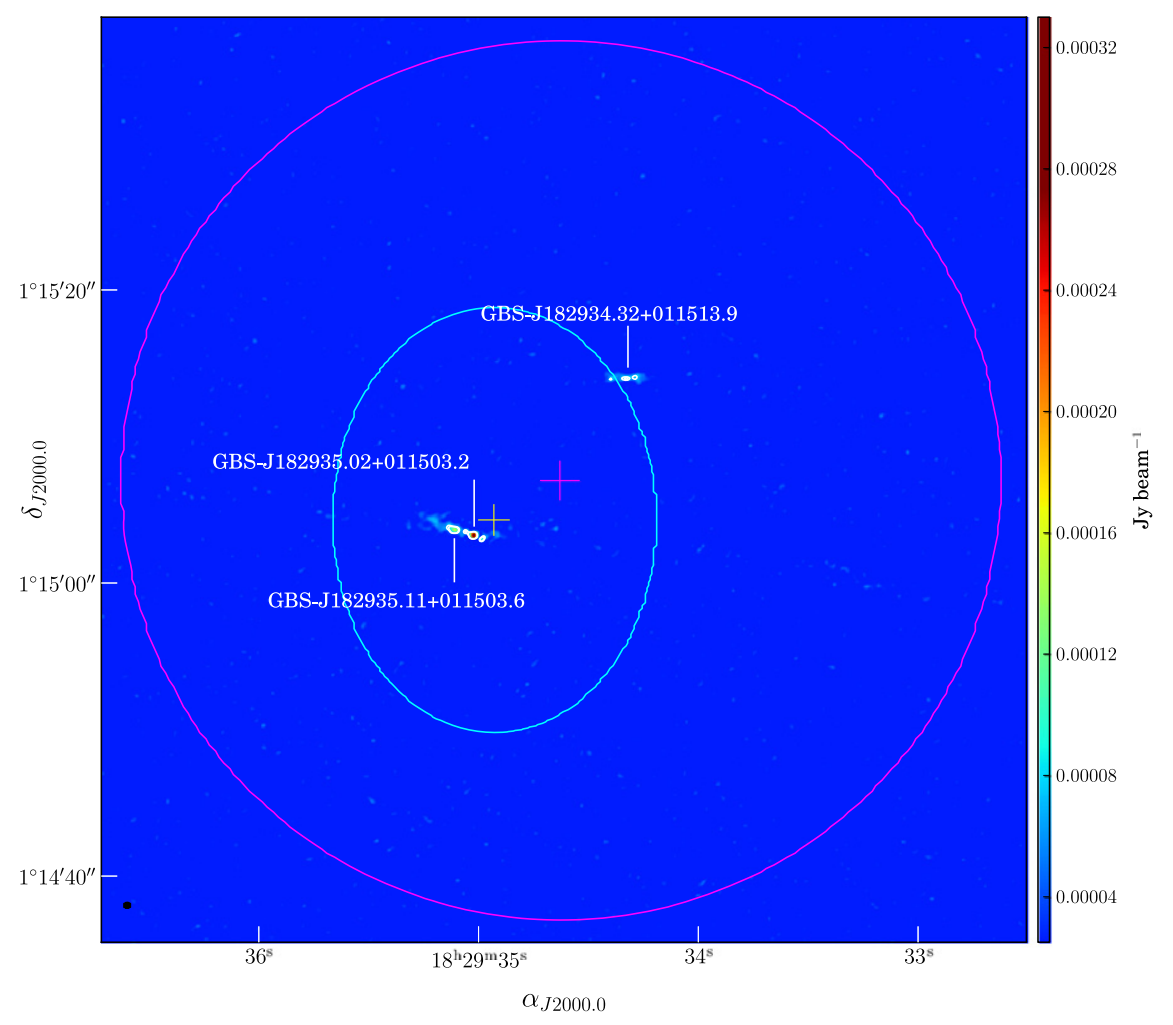

Figure $7.6 \mathrm{~cm}$ radio continuum image of three GBS-VLA sources in the Serpens molecular cloud. The $\theta_{\text {maj }} \times \theta_{\min }=0$ "' $40 \times 0 . " 39$, P.A. $=+96: 1$ synthesized beam is shown in the bottom left of the map. Contours are 5 and 6 times $15 \mu \mathrm{Jy}$ beam $^{-1}$, the rms noise of the image. The cyan ellipse indicates the size of the source NVSS $182934+011504\left(299^{\prime \prime} 0 \times 222^{\prime \prime} 1\right)$, from the catalog of Condon et al. (1998). The yellow cross marks, with error bars, the position of the NVSS source. The magenta circle indicates the size $\left(\sim 1^{\prime}\right)$ of the source DCE08-210 5, detected in the observations of AMI Consortium et al. (2012) at $1.8 \mathrm{~cm}$. The magenta cross marks, with error bars, the position of the $1.8 \mathrm{~cm}$ source.

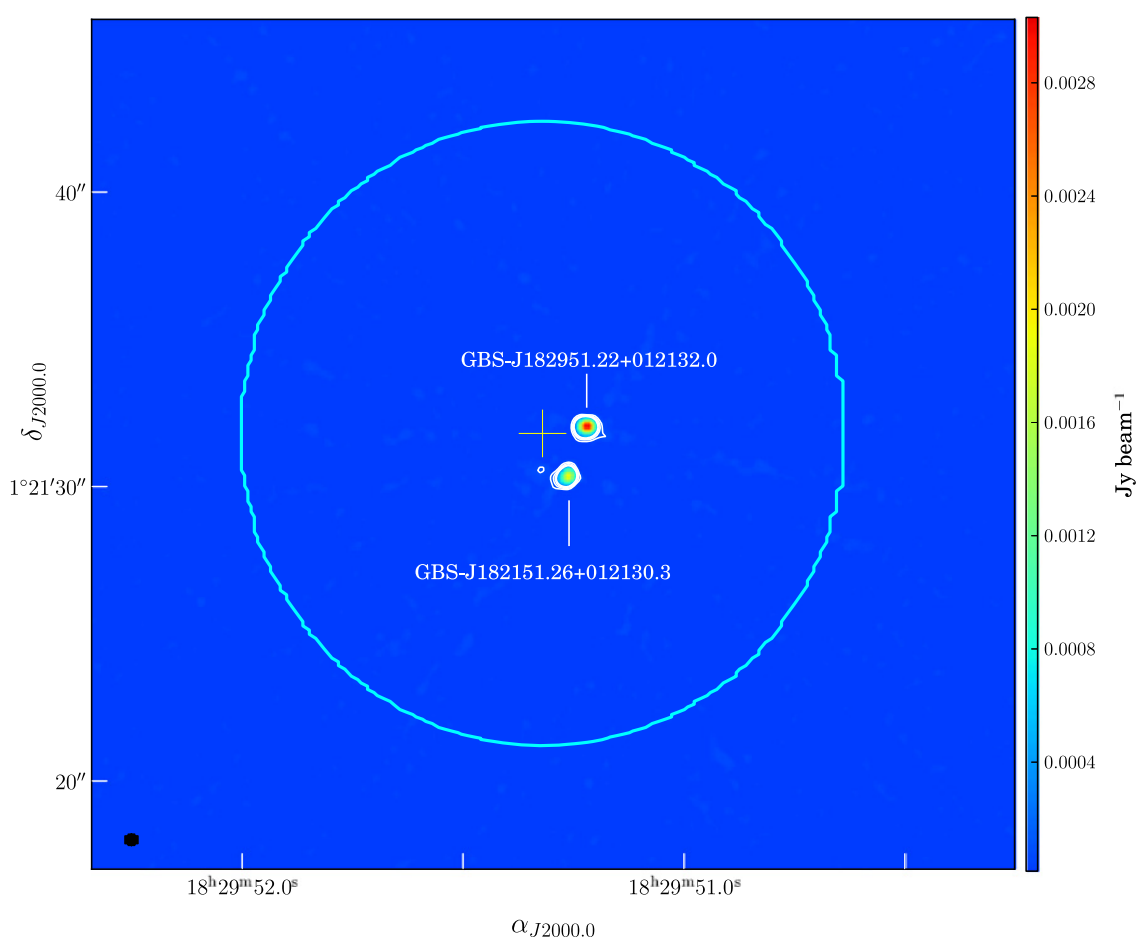

Figure $8.6 \mathrm{~cm}$ radio continuum image of two GBS-VLA sources in the Serpens molecular cloud. The $\theta_{\text {maj }} \times \theta_{\min }=0.40 \times 0$ " 39 , P.A. $=+96: 1$ synthesized beam is shown in the bottom left of the map. Contours are 5, 10,15, and 20 times $19 \mu \mathrm{Jy}$ beam $^{-1}$, the rms noise of the image. The cyan ellipse indicates the size of the source NVSS 182951+012131 $(21 . \prime 2 \times 20$ "'5), from the catalog of Condon et al. (1998). The yellow cross marks, with error bars, the position of the NVSS source. 


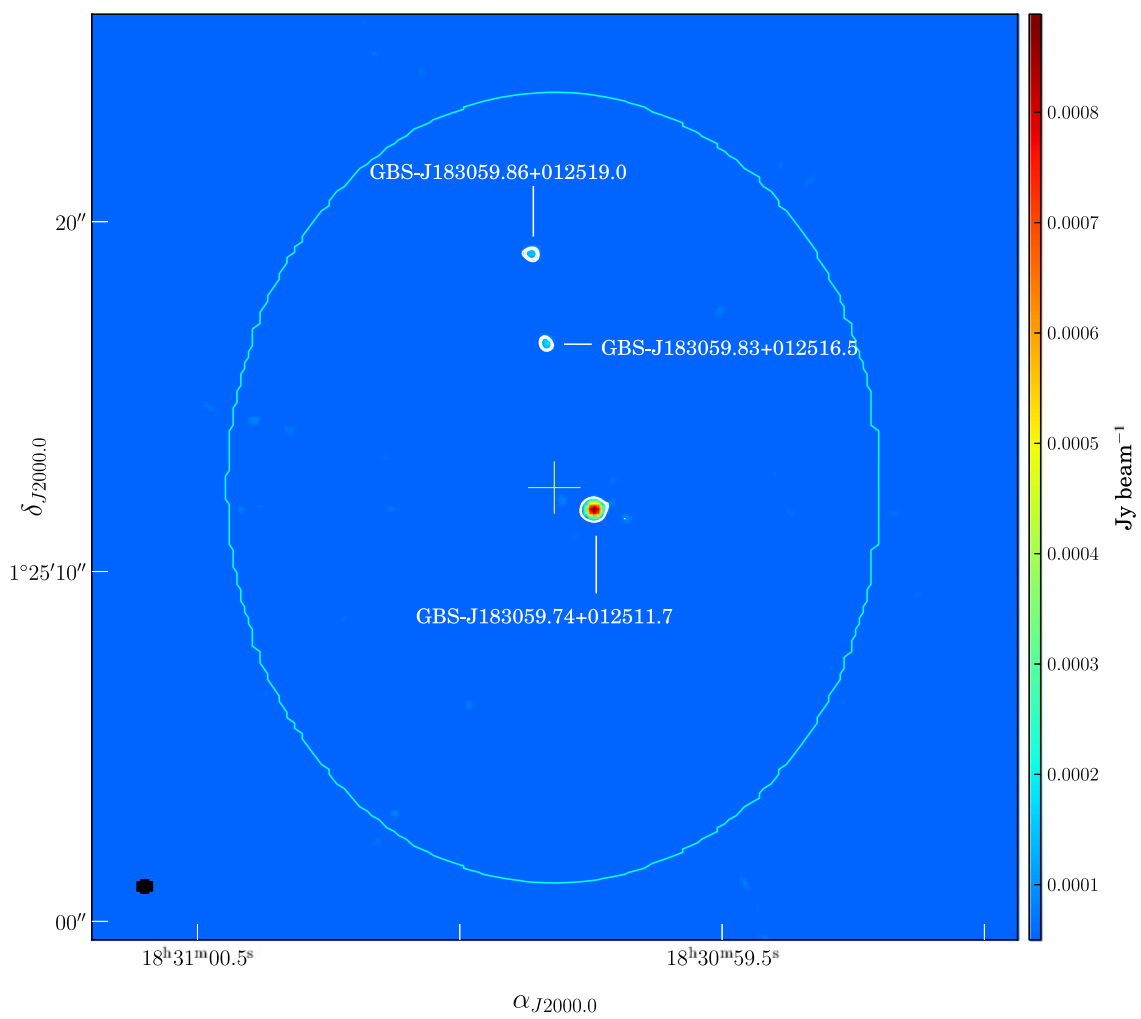

Figure $9.6 \mathrm{~cm}$ radio continuum image of three GBS-VLA sources in the Serpens molecular cloud. The $\theta_{\text {maj }} \times \theta_{\min }=00^{\prime \prime} 40 \times 0 . " 39$, P.A. $=+96: 1$ synthesized beam is shown in the bottom left of the map. Contours are 5, 6, and 7 times $17 \mu \mathrm{Jy}_{\text {beam }}{ }^{-1}$, the rms noise of the image. The cyan ellipse indicates the size of the source NVSS 183059+012512 (22"'6 × 18"'6), from the catalog of Condon et al. (1998). The yellow cross marks, with error bars, the position of the NVSS source.

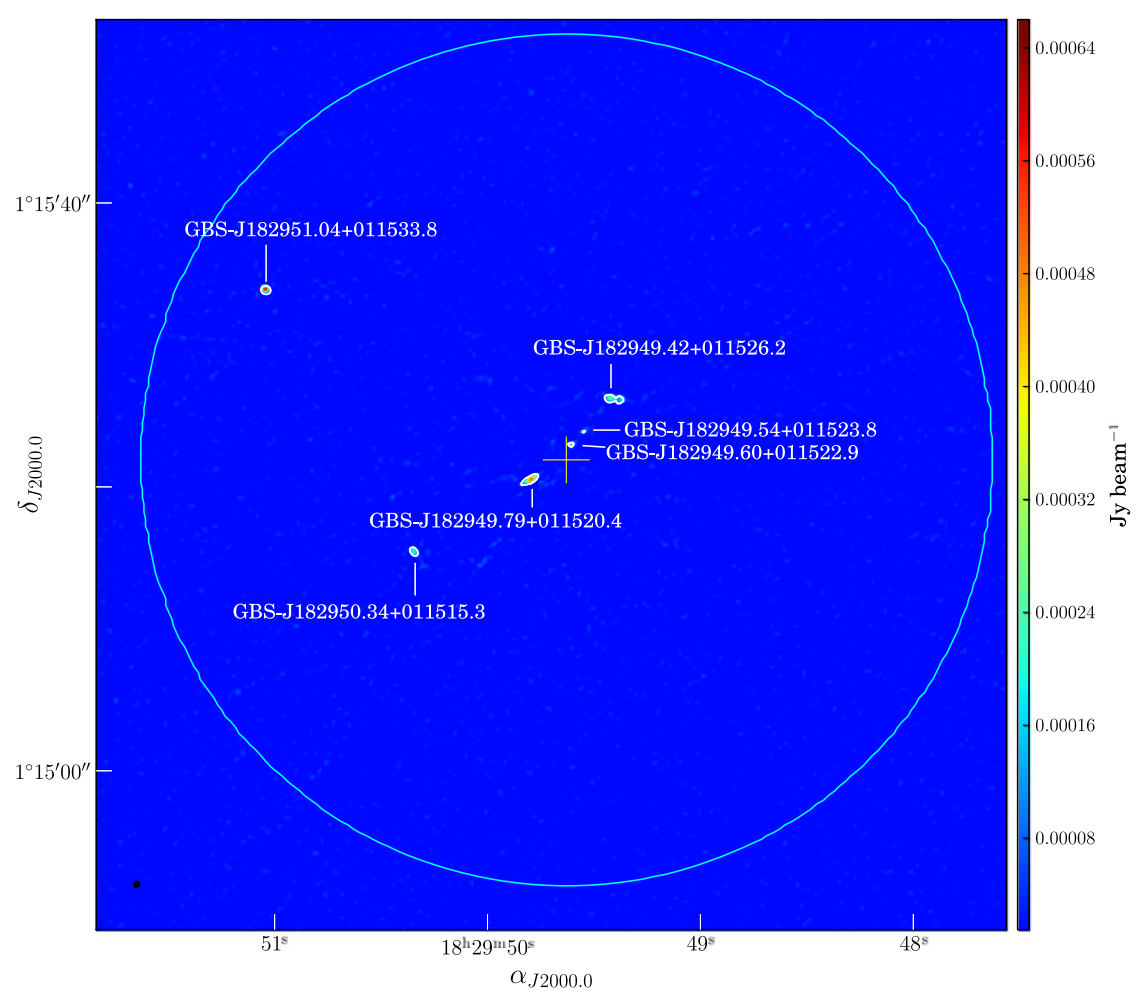

Figure $10.6 \mathrm{~cm}$ radio continuum image of six GBS-VLA sources in the Serpens molecular cloud. The $\theta_{\text {maj }} \times \theta_{\min }=00^{\prime \prime} 40 \times 0 . \prime 39$, P.A. $=+96^{\circ} 1$ synthesized beam is shown in the bottom left of the map. Contours are 5 and 6 times $16 \mu \mathrm{Jy}$ beam $^{-1}$, the rms noise of the image. The cyan circle indicates the size $\left(\sim 1^{\prime}\right)$ of the source DCE08-210 1, detected in the observations of AMI Consortium et al. (2012) at $1.8 \mathrm{~cm}$. The yellow cross marks, with error bars, the position of the $1.8 \mathrm{~cm}$ source. 
This work is supported by CONACyT, Mexico, and PAPIIT, UNAM. We thank M. Dunham and L. Allen for providing us with a list of known young stellar objects in the c2d-GB clouds prior to publication, and A. J. Maury for providing data of the Serpens South cluster. The National Radio Astronomy Observatory is operated by Associated Universities, Inc., under cooperative agreement with the National Science Foundation.

\section{APPENDIX}

For the 14 sources of the catalog by Rodríguez et al. (2010) that are detected in our observations we carried out an independent analysis of variability. In order to compare the flux densities of our observations at $7.5 \mathrm{GHZ}$ with the fluxes of Rodríguez et al. (2010) at $8.3 \mathrm{GHz}$, we extrapolated using the corresponding spectral indices (see Table A1). Unfortunately, in the paper of Rodríguez et al. (2010) no errors are given for the flux densities of the sources (because the flux densities reported are the average of the two epochs observed). Assuming a typical error of $20 \%$, we find that the flux densities of 3 of the 14 sources detected in both studies are consistent within $\pm 1 \sigma$. The remaining 11 sources show different flux densities for the two studies. Nine of the sources show a stronger flux density in the Rodríguez et al. (2010) paper, while the remaining two show a stronger flux density in this paper. This diversity seems to rule out that the sources are steady and that there is a systematic calibration error in one of the two studies (because then we would expect all sources to appear as brighter in one of the two studies). Finally, it should be stressed that in the Rodríguez et al. (2010) paper, which reported observations in two different epochs separated by 0.88 yr (2003 November 3 and 2004 September 18), 3 of these 11 sources were already reported as time variable. Since the present paper offers a larger time baseline (about a decade), we expect the sources to exhibit even more variability between the two studies.

\section{REFERENCES}

AMI Consortium, Scaife, A. M. M., Hatchell, J., et al. 2012, MNRAS, 420, 1019

André, P., Men'shchikov, A., Bontemps, S., et al. 2010, A\&A, 518, L102

Anglada, G., Villuendas, E., Estalella, R., et al. 1998, AJ, 116, 2953

Bontemps, S., André, P., Könyves, V., et al. 2010, A\&A, 518, L85

Cambrésy, L. 1999, A\&A, 345, 965

Casali, M. M., Eiroa, C., \& Duncan, W. D. 1993, A\&A, 275, 195

Condon, J. J., Cotton, W. D., Greisen, E. W., et al. 1998, AJ, 115, 1693

Crutcher, R. M., \& Chu, Y. H. 1982, Regions of Recent Star Formation, 93, 53

Cutri, R. M., Skrutskie, M. F., van Dyk, S., et al. 2003, VizieR Online Data Catalog, 2246, 0

Dame, T. M., Ungerechts, H., Cohen, R. S., et al. 1987, ApJ, 322, 706

Deller, A. T., Forbrich, J., \& Loinard, L. 2013, A\&A, 552, A51

Dobashi, K., Uehara, H., Kandori, R., et al. 2005, PASJ, 57, 1

Drake, S. A., Simon, T., \& Linsky, J. L. 1989, ApJS, 71, 905

Dulk, G. A. 1985, ARA\&A, 23, 169

Dunham, M. M., Arce, H. G., Allen, L. E., et al. 2013, AJ, 145, 94

Dunham, M. M., Crapsi, A., Evans, N. J., II, et al. 2008, ApJS, 179, 249

Dzib, S., Loinard, L., Mioduszewski, A. J., et al. 2010, ApJ, 718, 610

Dzib, S. A., Loinard, L., Mioduszewski, A. J., et al. 2013, ApJ, 775, 63

Dzib, S. A., Loinard, L., Rodríguez, L. F., \& Galli, P. 2014, ApJ, 788, 162 Eiroa, C., \& Casali, M. M. 1992, A\&A, 262, 468

Eiroa, C., Djupvik, A. A., \& Casali, M. M. 2008, in Handbook of Star Forming Regions, Vol. 2, The Southern Sky ASP Monograph Publications, Vol. 5, ed. B. Reipurth (San Francisco, CA: ASP), 693

Eiroa, C., Torrelles, J. M., Curiel, S., \& Djupvik, A. A. 2005, AJ, 130, 643

Enoch, M. L., Evans, N. J., II, Sargent, A. I., \& Glenn, J. 2009, ApJ, 692, 973
Estalella, R., Anglada, G., Rodriguez, L. F., \& Garay, G. 1991, ApJ, 371, 626 Evans, N. J., II, Dunham, M. M., Jørgensen, J. K., et al. 2009, ApJS, 181, 321 Feigelson, E. D., Carkner, L., \& Wilking, B. A. 1998, ApJL, 494, L215 Feigelson, E. D., \& Montmerle, T. 1985, ApJL, 289, L19

Felli, M., Taylor, G. B., Neckel, T., \& Staude, H. J. 1998, A\&A, 329, 243

Fernández-López, M., Arce, H. G., Looney, L., et al. 2014, ApJL, 790, LL19

Forbrich, J., Massi, M., Ros, E., Brunthaler, A., \& Menten, K. M. 2007, A\&A, 469,985

Garay, G., Ramirez, S., Rodriguez, L. F., Curiel, S., \& Torrelles, J. M. 1996, ApJ, 459, 193

Giardino, G., Favata, F., Micela, G., Sciortino, S., \& Winston, E. 2007, A\&A, 463,275

Giovannetti, P., Caux, E., Nadeau, D., \& Monin, J.-L. 1998, A\&A, 330, 990

Gómez, Y., Rodríguez, L. F., \& Garay, G. 2000, ApJ, 531, 861

Gomez de Castro, A. I., Eiroa, C., \& Lenzen, R. 1988, A\&A, 201, 299

Goss, W. M., \& Shaver, P. A. 1970, AuJPA, 14, 1

Güdel, M., \& Benz, A. O. 1993, ApJL, 405, L63

Güdel, M., Schmitt, J. H. M. M., Bookbinder, J. A., \& Fleming, T. A. 1993, ApJ, 415, 236

Gutermuth, R. A., Bourke, T. L., Allen, L. E., et al. 2008, ApJL, 673, L151

Harvey, P., Merín, B., Huard, T. L., et al. 2007a, ApJ, 663, 1149

Harvey, P. M., Chapman, N., Lai, S.-P., et al. 2006, ApJ, 644, 307

Harvey, P. M., Rebull, L. M., Brooke, T., et al. 2007b, ApJ, 663, 1139

Hovatta, T., Nieppola, E., Tornikoski, M., et al. 2008, A\&A, 485, 51

Hovatta, T., Tornikoski, M., Lainela, M., et al. 2007, A\&A, 469, 899

Hughes, V. A. 1988, ApJ, 333, 788

Hughes, V. A. 1991, ApJ, 383, 280

Hughes, V. A., Cohen, R. J., \& Garrington, S. 1995, MNRAS, 272, 469

Hurt, R. L., \& Barsony, M. 1996, ApJL, 460, L45

Kaas, A. A. 1999 , AJ, 118,558

Kaas, A. A., Olofsson, G., Bontemps, S., et al. 2004, A\&A, 421, 623

King, D. J., Scarrott, S. M., \& Taylor, K. N. R. 1983, MNRAS, 202, 1087

Könyves, V., André, P., Men'shchikov, A., et al. 2010, A\&A, 518, L106

Kuhn, M. A., Getman, K. V., Feigelson, E. D., et al. 2010, ApJ, 725, 2485

Kurtz, S. 2005, Massive Star Birth: A Crossroads of Astrophysics, 227, 111

Loinard, L. 2013, in IAU Symp. 289, Advancing the Physics of Cosmic Distances, ed. R. de Gris \& G. Bono (Cambridge: Cambridge Univ. Press), 36

Loren, R. B., Evans, N. J., II, \& Knapp, G. R. 1979, ApJ, 234, 932

Lovell, J. E. J., Rickett, B. J., Macquart, J.-P., et al. 2008, ApJ, 689, 108

Maury, A. J., André, P., Men'shchikov, A., Könyves, V., \& Bontemps, S. 2011, A\&A, 535, A77

Molinari, S., Brand, J., Cesaroni, R., \& Palla, F. 1996, A\&A, 308, 573

Oliveira, I., Pontoppidan, K. M., Merín, B., et al. 2010, ApJ, 714, 778

Panagia, N., \& Felli, M. 1975, A\&A, 39, 1

Perrot, C. A., \& Grenier, I. A. 2003, A\&A, 404, 519

Preibisch, T. 1998, A\&A, 338, L25

Preibisch, T. 1999, A\&A, 345, 583

Preibisch, T. 2003, A\&A, 410, 951

Preibisch, T. 2004, A\&A, 428, 569

Predehl, P., \& Schmitt, J. H. M. M. 1995, A\&A, 293, 889

Rodriguez, L. F., González, R. F., Montes, G., et al. 2012, ApJ, 755, 152

Rodriguez, L. F., Marti, J., Canto, J., Moran, J. M., \& Curiel, S. 1993, RMxAA, 25, 23

Rodriguez, L. F., Myers, P. C., Cruz-Gonzalez, I., \& Terebey, S. 1989, ApJ, 347,461

Rodríguez, L. F., Rodney, S. A., \& Reipurth, B. 2010, AJ, 140, 968

Shuping, R. Y., Vacca, W. D., Kassis, M., \& Yu, K. C. 2012, AJ, 144, 116

Smith, J., Bentley, A., Castelaz, M., et al. 1985, ApJ, 291, 571

Smith, K., Güdel, M., \& Benz, A. O. 1999, A\&A, 349, 475

Sogawa, H., Tamura, M., Gatley, I., \& Merrill, K. M. 1997, AJ, 113, 1057

Straižys, V., Černis, K., \& Bartašiūte, S. 1996, BaltA, 5, 125

Strom, S. E., Grasdalen, G. L., \& Strom, K. M. 1974, ApJ, 191, 111

Strom, S. E., Vrba, F. J., \& Strom, K. M. 1976, AJ, 81, 638

Testi, L., \& Sargent, A. I. 1998, ApJL, 508, L91

Torrelles, J. M., Verdes-Montenegro, L., Ho, P. T. P., Rodriguez, L. F., \& Canto, J. 1989, ApJ, 346, 756

Vallee, J. P., \& MacLeod, J. M. 1994, AJ, 108, 998

Warren-Smith, R. F., Draper, P. W., \& Scarrott, S. M. 1987, MNRAS, 227, 749

Westerhout, G. 1958, BAN, 14, 215

Winston, E., Megeath, S. T., Wolk, S. J., et al. 2010, AJ, 140, 266

Worden, S. P., \& Grasdalen, G. L. 1974, A\&A, 34, 37

Wright, E. L., Eisenhardt, P. R. M., Mainzer, A. K., et al. 2010, AJ, 140,1868 